\title{
The xerobranching response represses lateral root formation when roots are not in contact with water
}

\author{
Beata Orman-Ligeza ${ }^{1,2,3,14,16}$, Emily C. Morris ${ }^{4,5,16}$, Boris Parizot ${ }^{2,3}$, Tristan \\ Lavigne $^{1}$, Aurelie Babé ${ }^{1}$, Aleksander Ligeza ${ }^{1}$, Stephanie Klein 6 , \\ Craig Sturrock ${ }^{4,5}$, Wei Xuan ${ }^{7}$, Ondřey Novák ${ }^{8,9}$, Karin Ljung ${ }^{8}$, Maria \\ Fernandez ${ }^{10}$, Pedro L. Rodriguez ${ }^{10}$, Ian C. Dodd ${ }^{11}$, Ive De Smet ${ }^{2,3,4,5}$, Francois \\ Chaumont ${ }^{12}$, Henri Batoko ${ }^{12}$, Claire Périlleux ${ }^{13}$, Jonathan P. Lynch ${ }^{4,5,6}$, \\ Malcolm J. Bennett ${ }^{4,5}$, Tom Beeckman ${ }^{2,3}$ and Xavier Draye ${ }^{1,15,{ }^{*}}$
}

${ }^{1}$ Earth and Life Institute, Université catholique de Louvain, B-1348 Louvain-la-Neuve, Belgium.

2Department of Plant Biotechnology and Bioinformatics, Ghent University, B-9052 Ghent, Belgium.

${ }^{3}$ Department of Plant Systems Biology, VIB, B-9052 Ghent, Belgium.

${ }^{4}$ Division of Plant and Crop Sciences, School of Biosciences, University of Nottingham, Leicestershire, LE12 5RD, United Kingdom.

${ }^{5}$ Centre for Plant Integrative Biology, School of Biosciences, University of Nottingham, Sutton Bonington, LE12 5RD, United Kingdom.

${ }^{6}$ Department of Plant Science, The Pennsylvania State University, University Park, PA, USA 16802

${ }^{7}$ State Key Laboratory of Crop Genetics and Germplasm Enhancement, MOA Key Laboratory of Plant Nutrition and Fertilization in Lower-Middle Reaches of the Yangtze River, Nanjing Agricultural University, Nanjing 210095, PR China

${ }^{8}$ Department of Forest Genetics and Plant Physiology, Umeå Plant Science Centre, SE-901 83 Umeå, Sweden.

${ }^{9}$ Centre of the Region Haná for Biotechnological and Agricultural Research, Laboratory of Growth Regulators, Palacký University and Institute of Experimental Botany AS CR, Šlechtitelů 11, CZ-78371 Olomouc, Czech Republic.

${ }^{10}$ Instituto de Biología Molecular y Celular de Plantas, Consejo Superior de Investigaciones CientificasUniversidad Politecnica de Valencia, ES-46022 Valencia, Spain

${ }^{11}$ The Lancaster Environment Centre, Lancaster University, LA1 4YQ, United Kingdom.

${ }^{12}$ Louvain Institute for Biomolecular Sciences and Technologies, Université catholique de Louvain, B-1348 Louvain-la-Neuve, Belgium.

${ }^{13}$ InBioS - PhytoSYSTEMS, Laboratory of Plant Physiology, University of Liège, B-4000 Liège, Belgium.

${ }^{14}$ Current address: National Institute of Agricultural Biology, Huntingdon Road, Cambridge CB3 OLE, United Kingdom

${ }^{15}$ Lead contact

${ }^{16}$ These authors contributed equally 
*Correspondence: xavier.draye@uclouvain.be 


\section{Summary}

Efficient soil exploration by roots represents an important target for crop improvement and food security [1, 2]. Lateral root (LR) formation is a key trait for optimising soil foraging for crucial resources such as water and nutrients. Here, we report an adaptive response termed xerobranching, exhibited by cereal roots, that represses branching when root tips are not in contact with wet soil. Non-invasive $\mathrm{X}$-ray microCT imaging revealed that cereal roots rapidly repress $L R$ formation as they enter an air space within a soil profile and are no longer in contact with water. Transcript profiling of cereal root tips revealed that transient water deficit triggers the abscisic acid ( $A B A)$ response pathway. In agreement with this, exogenous $A B A$ treatment can mimic repression of $L R$ formation under transient water deficit. Genetic analysis in Arabidopsis revealed that $A B A$ repression of $L R$ formation requires the PYR/PYL/RCARdependent signalling pathway. Our findings suggest that $A B A$ acts as the key signal regulating xerobranching. We conclude that this new ABA-dependent adaptive mechanism allows roots to rapidly respond to changes in water availability in their local micro-environment and to use internal resources efficiently.

\section{Key words}

Lateral roots, soil air spaces, water deficit, root system architecture, abscisic acid, auxin, cereal crops, Arabidopsis 


\section{Results and Discussion}

\section{Root branching is repressed in soil air spaces}

Plants depend on soil resources, like water and nutrients, that are often distributed in highly heterogeneous patterns. To optimise foraging, plants have evolved several root developmental responses to soil heterogeneity. Root system architecture is therefore highly plastic and shaped by many environmental signals [3-7]. In particular, root development responds to the soil physical structure, which consists of aggregates and pores of varying sizes. It has been demonstrated recently that roots growing down the side of a macropore will only induce LR formation along the arc of their circumference in contact with wet soil aggregates. This auxin-dependent response has been termed hydropatterning [7]. However, when roots grow across a large macropore and loose contact with soil aggregates, the root segments within the macropore are likely to be exposed to air and experience a local transient water deficit. Indeed, large macropores have a reduced water retention capacity and loose water rapidly after precipitation events. Surprisingly, it is currently unclear how roots respond to such transient events.

X-ray microscale computed tomography (microCT) was employed to noninvasively image maize (Zea mays) $(n=5)$ and barley (Hordeum vulgare) $(n=$ 3) roots growing across a large macropore. Seedling roots initially grew through $4 \mathrm{~cm}$ of soil, then entered a $2 \mathrm{~cm}$ air space and later re-entered $4 \mathrm{~cm}$ of soil. MicroCT imaging revealed that LR branching was highly responsive to external environmental conditions (Figures $1 A$ and $B$ ). We observed that LR formation occurs along the roots growing within the soil, is almost completely repressed in root segments growing through the air-filled macropore and is triggered again when roots re-enter the soil (Figures 1A and B; Video S1). In contrast, when the air space was filled with water, maize roots continued to form LRs (compare air versus water filled spaces in Figures $S 1 A$ and $B ; n=3$ for each treatment). Hence, LR branching in maize and barley seedling roots appears to be highly dependent on external contact with the soil-water matrix.

We next addressed whether LR branching was repressed at organ initiation and/or emergence stages when roots were growing through macropores. 
Radial sections were generated through cereal root segments grown in our soilmacropore-soil experimental system using a vibratome in combination with confocal microscopy (3 plants sectioned for both maize and barley creating 54 and 57 images respectively) (Figures $1 \mathrm{C}$ to $\mathrm{H}$ ). This radial sectioning approach suggested that LR branching was repressed at organ initiation, before stage IV as no primodria were visible, in root segments growing through the macropore (Figures 1D and G) $[5,8]$. We validated our vibratome-confocal imaging results by sectioning maize roots at a higher density of radial sections using a laser ablation tomography (LAT) based approach (4 plants sectioned creating 187 images) (Figure S1C). LAT simultaneously sections and images the surface of samples every $1 \mathrm{~mm}$ along root segments. LAT sectioning confirmed that LR branching was arrested at the organ initiation stage, before stage IV primodia, as almost no new primordia were visible in root segments grown within the macropore, contrasting with samples grown in contact with soil (Figure S1C). Our soil-macropore-soil experiment yields the same type of LR repression observed in aeroponically grown roots exposed to transient water deficit (WD), which leads to the formation of a LR repression zone [9]. We suggest that the loss of soil contact and/or the drop of matric water potential is perceived by the growing root, causing repression of LR formation.

\section{Transient water deficit induces ABA and auxin responsive genes in barley roots}

We investigated the gene expression responses triggered in root tips by exposure to a transient WD. Since the macropore traversal system is not amenable to sampling for molecular studies, and given the phenotypic similarity between macropore traversal and transient WD in aeroponics, we used the latter system to analyse the molecular mechanisms and signal(s) associated with LR repression in barley and maize (Figure S2A). Barley roots exposed to 2 to 6 hour long water deficit treatment in aeroponics were transcript profiled using the Barley1 GeneChip Genome Arrays (22,782 contigs) on segments of the primary seminal root containing the LR repression window delineated by Babe et al., 2012 [9]. 755 genes exhibited a significant treatment $x$ time interaction over the WD treatment (ANOVA2, $p$-value $\leq 0.01, F C \geq 1.5$, FDR $p$ value $\leq 0.01$ ) (Figure $2 A$ and Data S1). We found a significant enrichment of 
genes involved in cytokinin, ABA, auxin, salicylic acid and jasmonic acid hormone pathways (respectively 2.1, 2.3, 2.5, 2.8 and 4.6, p-value $\leq 0.01$ ) (Figure 2B). We have performed RT-qPCR validation for a bZIP transcription factor (termed Contig_10961; Figures S2B to D). Due to the well known roles of $A B A$ in drought signalling we focused on its role in xerobranching [10].

Arabidopsis orthologs were available for 506 out of the 755 cereal sequences. We could therefore compare the cereal gene list with different datasets related to LR formation in Arabidopsis [11-14] (Data S1). The highest gene enrichments were observed in datasets generated after treatment with auxin-related signalling molecules naxillin and indole-3-butyric acid (IBA) (respectively 2.6x and $2.7 x$, $p$-value $\leq 0.01$ ), which modify priming of pericycle cells prior to $L R$ initiation. We also noticed a significant but lower enrichment for the two datasets related to LR initiation itself $(1.5 x$ for both SLR dependant $L R$ initiation and xylem pole pericycle specific LR initiation, $p$-value $\leq 0.01)$. The above microarray experiments were designed to target transcriptional events that occur during early stages of LR formation in Arabidopsis, therefore we hypothesize that the transient WD episode interferes with the LR initiation stage or earlier.

\section{ABA treatment mimics the root branching response to transient WD}

To assess the role of $A B A$ as a regulator of $L R$ repression, we first quantified ABA levels in barley roots during WD. WD triggered a $\sim 4$ fold increase in active pools of $A B A$ in root tissue, compared to control plants, within 4 hours of treatment (Figure 2C). Interestingly, ABA levels in aeroponically grown sunflower roots following a WD treatment were also markedly higher compared to unstressed controls [15].

To test the functional importance of $A B A$ accumulation in roots, we exposed barley and maize seedling roots to 6 hours of transient $50 \mu \mathrm{m}$ ABA treatment in the aeroponics system. Clear LR repression zones appeared along the root of ABA-treated barley and maize seedlings (Figures 2D and E) and their localisation was in complete agreement with that of repression zones formed under transient WD [9]. The effect of transient ABA application was also assessed on agar plates with barley to clarify the same response seen in 
hydroponics is also present on agar plates. (Figure S3). In agreement with cereal experiments, Arabidopsis seedlings grown on agar plates treated with ABA for 2 days lacked LRs in the root segment that had been formed in the presence of $A B A$ (Figures $3 A$ to $E$ ). The increase of $A B A$ concentration in the root appears therefore to be able to mimic LR repression induced by transient WD (and presumably by soil macropores) in cereals and Arabidopsis.

In Arabidopsis, ABA is perceived by members of a redundant family of receptors encoded by $13 P Y R / P Y L / R C A R$ genes [16]. We exposed Arabidopsis seedlings, 5 days post germination (dpg), of Col-0, higher-order ABA receptor mutants pyr1 pyl1 pyl2 pyl4 pyl5 pyl8 pyl9 (pyr/pyl 1124589), pyr/pyl 1124578 and pyr/pyl 112458 to a transient $30 \mu \mathrm{M}$ ABA treatment as described above. Repression zones were observed in the root segments that grew in the presence of $\mathrm{ABA}$ in Col-0, whereas all pyr/pyl mutants were resistant to ABA treatment and formed LR (Figures $3 F$ to $H$ ). We therefore concluded that the $L R$ repression event is mediated by an ABA signaling module downstream of ABA perception by PYR/PYL receptors in Arabidopsis.

\section{ABA represses LR development prior to organ initiation}

Based on the above results, the transient ABA treatment was used as an experimental assay to explore the mechanisms of LR repression in cereals and Arabidopsis. In the three species analyzed, the sharpness of the repression zone boundaries suggested that the repression targets a specific developmental window during LR formation. In barley and maize control plants, the root segment spanning the region where the repression zone appears in ABA-treated plants contains ca. 10 emerged LR $(9.5 \pm 1.7, n=14)$ [9]. In the presence of $A B A$, no LR primordia were observed in the repression zone, with a few exceptions where the repression zone contained 1-3 primordia arrested at the first asymmetric cell division stage in barley, or at more advanced stages in maize ( $n=20$ for each species). In Arabidopsis control seedlings, the root segment corresponding to the region where the repression zone appears in treated plants contains ca. 4 emerged LRs $(4.12 \pm 0.9, \mathrm{n}=10)$. In the presence of 5 and $10 \mu \mathrm{M}$ ABA, the repression zone either contained no LR primordia (5/12 and 4/11, respectively) or 1 - 2 LRs blocked between stage $V$ and soon 
after emergence. At 30 and $50 \mu \mathrm{M} \mathrm{ABA}$, the repression zone did not contain any visible $L R$ primordia ( $n=12$ and $n=15$, respectively), except for $230 \mu M$ treated seedlings for which the repression zone contained $1 \mathrm{LR}$ primordium arrested at stage I (2/15). The transient exposure to ABA therefore appears to interfere with LR initiation stages, before the first formative divisions.

In these transient treatments, the repression zone is slightly offset from the segment that was developed during the treatment. The proximal side of the repression zone comprises a short segment (d1) that was formed before the treatment onset, but in which LR formation was nevertheless inhibited by ABA. Interestingly the length of $\mathrm{d} 1$ increases with higher $A B A$ concentrations (Figure $3 D$ ). This suggests a higher dose of $A B A$ is able to have a more severe effect on the root that has already developed. This increase in $\mathrm{d} 1$ is why the slow growth rates seen at 30 and $50 \mu \mathrm{m}$ do not result in shorter repression zones (Figures $3 \mathrm{~B}$ and $\mathrm{C}$ ). The distal side of the repression zone is followed by a short segment (d2) which was formed during the ABA treatment, but in which LR formation was not inhibited by ABA. As a consequence LR number counted in Col-0 in the root portion grown on ABA is higher than 0 because one or multiple LRs emerge in d2 (Figure 3H).

The distance from the root tip correlates well with the developmental stages of LR formation, therefore the length of $d 1$ indicates which stages could have been present before the treatment and affected by the ABA treatment. Similarly, the developmental stage reached in $\mathrm{d} 2$ are still capable of forming $L R$ after release of $A B A$. The developmental window targeted by the ABA treatment is therefore the root zone located between $\mathrm{d} 1$ and $\mathrm{d} 2$ from the root tip. The offsets $\mathrm{d} 1$ and d2 were measured in barley and Arabidopsis as described in [9] and (Figure $3 A$ ). In barley, d1 and d2 offsets were, respectively, $14.4 \pm 0.11$ and $12 \pm 0.32$ $\mathrm{mm}$, and delimit a region where LR root primordia are at stage I or before. In Arabidopsis, d1 and d2 values at 5 and $10 \mu \mathrm{M}$ ABA were both close to $1 \mathrm{~mm}(\mathrm{n}$ = 12 for each concentration) (Figure $3 \mathrm{D}$ and $\mathrm{E}$ ), which falls within the $0.2-1.2$ $\mathrm{mm}$ region near the root tip where LR priming occurs [17-20]. At both 30 and $50 \mu \mathrm{M}$ ABA, d1 and d2 were respectively close to 3.4 and $1.1 \mathrm{~mm}$, which spans the transition from founder cell specification and primordium initiation that occurs $4.2 \mathrm{~mm}$ from the tip [17]. The evidence obtained in barley and 
Arabidopsis indicate that the ABA repression occurs before the first asymmetric division and, according to our Arabidopsis results, at or before LR founder cell specification.

\section{ABA affects prebranch site formation}

To test whether ABA repression occurs at or before LR founder cell specification, we monitored the activity of the Arabidopsis DR5:Luciferase reporter which has been shown to oscillate close behind the root tip and demarks the pre-branch sites in which the LR founder cells are located [21]. When $5 \mathrm{dpg}$ Arabidopsis seedlings were exposed for 2 days to increasing levels of $A B A$, a concentration-dependent decrease of the number of primed sites was observed (11.4 \pm 2.8 mock treatment, $7.8 \pm 2.91 \mu \mathrm{M} \mathrm{ABA}, 4.2 \pm 2.83 \mu \mathrm{M}$ ABA, $4.3 \pm 2.810 \mu \mathrm{M}$ ABA and $1.0 \pm 1.430 \mu \mathrm{M} A B A, n=13)$. This suggested that transient $A B A$ treatment affects the formation of pre-branch sites in close proximity to the root tip. The DR5:Luciferase oscillations which were observed at $0.5 \mu \mathrm{M}$ ABA [22] were blocked at $30 \mu \mathrm{M}$ ABA (Figure 4A and Video S2). By monitoring DR5:rev:3xVenus-N7 fluorescence at $30 \mu \mathrm{M} \mathrm{ABA}$, we also showed that the auxin response decreased in initiated sites and was lacking in putative primed sites (Figure S4A and Video S2). Increased levels of ABA therefore appears to attenuate the oscillatory auxin response network located in the basal meristem.

To relate this observation in Arabidopsis to our model cereals we exposed maize seedlings expressing a DR5:RFP transgene to 4 hour long $50 \mu \mathrm{M} A B A$ treatment in aeroponics. We observed that the fluorescence signal decreased in the root tip (Figure S4B). Taken together, these results in maize and Arabidopsis suggests that transient ABA treatment represses the formation of new primordia by disrupting auxin responses during early LR initiation.

\section{Transient ABA treatment decreases free IAA levels in roots}

Given the promotive role of auxin during LR formation [23-25], we investigated whether co-treatment with this hormone could reverse ABA-mediated LR repression (Figures $4 \mathrm{~B}$ and $\mathrm{C}$ ). To test this, $5 \mathrm{dpg}$ barley seedlings were exposed for 1 hour to $75 \mu \mathrm{M}$ 1-Naphthaleneacetic acid (NAA, $n=18$ ), indole-3- 
acetic acid (IAA, $n=19)$ or 2,4-Dichlorophenoxyacetic acid (2,4-D, $n=15)$ prior to a 6 hour long $50 \mu \mathrm{M}$ ABA treatment in aeroponics $(n=14$ no treatment control, $\mathrm{n}=17$ ABA treatment control) (Figures 4B and C). A dosage series from 1 to $100 \mu \mathrm{M}$ was completed and the results from a $75 \mu \mathrm{M}$ application is reported here as a representative result (Data S2). Pre-treatments with the synthetic and natural forms of auxin, NAA and IAA, prevented LR repression fully and partially, respectively. Interestingly, post-treatment of barley seedlings with the same auxins did not restore LR formation (Figure S4C). The inability of auxin posttreatment to restore LR formation suggests that barley pericycle cells exposed to a transient ABA treatment irreversibly loose their competence to respond to auxin and to engage in LR formation.

Finally, we investigated whether the transient $A B A$ treatment decreases free IAA levels in maize. IAA profiling was performed on $0.5 \mathrm{~cm}$ long segments along the $2 \mathrm{~cm}$ long distal region of maize roots following a treatment with ABA during 2 to 6 hours. The profiling focused on free IAA, several IAA precursors (Trp (Tryptophan), Tra (Tryptamine), IpyA (Indole-3-pyruvic acid)), products of IAA inactivation (oxidative intermediate oxIAA (2-oxindole-3-acid acid) and IAA conjugates with Asp (Aspartate) and Glu (Glutamate) [26, 27]. Despite a transient increase of free IAA between 2 and 4 hours of treatment in the distal segments, the ABA treatment progressively decreased free IAA levels down to $80 \%$ of the control in $0.5-2 \mathrm{~cm}$ segment after 6 hours of treatment (Figure 4D). The amount of IAA conjugates increased after 2 hours of treatment, in parallel to the free IAA decrease in the proximal region, with only a minor impact on auxin biosynthesis (Figures 4E and F). We can therefore conclude that ABA leads to a decrease of auxin levels above the root apical meristem in maize, consistent with the above observation in barley that auxin pre-treatment prevents the repressive effect of ABA on LR initiation.

\section{Conclusions}

Roots are known to exhibit a high level of developmental plasticity in response to their soil environment. However, how roots sense and react to transient variations in water availability has not been addressed in detail to date, most probably because soil water is generally considered as a resource subject to progressive depletion. Our study reveals that LR formation is repressed at an 
early stage when roots grow through air-filled soil macropores (Figure 1). We demonstrate that, in the analogous transient WD assay [9], the ABA response pathway is involved in LR repression (Figure 4G). This LR adaptive response appears to be specific to transient treatments as it does not occur under prolonged WD treatments [28-30], nor under prolonged exposure to ABA [29]. It is also different from the earlier described drought rhizogenesis that is the result of a progressive drought stress. In the latter case, LR primordia are formed at a normal density and remain blocked at the meristem activation phase. More specifically, a long term exposure to ABA inhibits LR meristem activation by promoting quiescence of $\mathrm{QC}$ cells in a reversible fashion and does not affect early stages of LR formation [29, 31, 32]. Hence, our observation of the repression of LR formation early after the onset of transient WD or ABA treatment appears to be distinct from the well described effects of ABA under prolonged WD [28, 33, 34].

Based on our results, we propose that xerobranching is the outcome of an ABAdependent regulatory mechanism operating in a narrow developmental window, which interfers with the constituative branching mechanism in plant roots. We hypothesise that $A B A$ levels accumulate as the root tip experiences a transient WD, disrupting the auxin response and repressing LR priming. Our results suggest that $A B A$ reduces auxin accumulation. Whether this is a direct consequence of altered auxin metabolism, or a secondary effect caused by altering the auxin signalling machinary, is not clear. When the root tip reconnects with soil aggregates providing water, ABA gets diluted and the repression of LR priming is lifted. Such a mechanism would indeed switch the repression on and off whenever the root segment is exposed to air or wet soil, respectively, thereby ensuring the perfect match of the repression zone with macropore boundaries.

The apparent phenotypic similarily between the xerobranching and hydropatterning responses would suggest that the former is an extreme manifestation of the latter, which occurs when the whole root circumference looses contact with soil moisture. However, our experimental results indicate that $A B A$ is a key signal invovled in xerobranching whilst Bao et al., 2014 observed that $A B A$ signalling does not play a regulatory role in hydropatterning. 
[7]. Whilst the mechanistic link between these two responses is not clear, the striking effect of water availability on root branching is clear and shows that plants have evolved sophisticated signalling repsonses to navigate the complex soil system.

\section{Aknowledgements}

This work was supported by the Fund for Research Training in Industry and Agriculture (FRIA, FRS-FNRS) for PhD grants to B.O.-L., A.B. and T.L., Biological and Biotechnology Science Research Council (BBSRC) Doctoral Training studentship to E.C.M, FWO Vlaanderen (G0273.13N) to B.O.-L., FNRS (CC T.L. and B.O.-L.) to X.D., EU EuRoot (EU-FP7, Grant Agreement No 289300.27) to X.D., F.C. and M.J.B., DROPs (EU-FP7, Grant Agreement $\mathrm{N}^{\circ}$ 244374) to X.D., F.C. and A.L., IAP (grant IAP7/29) to B.O.-L., B.P., T.B., M.J.B., F.C. and X.D., the Communauté française de Belgique (ARC 11/16036) to B.O.-L., F.C., H.B. and X.D., BBSRC David Phillips Fellowship (BB_BB/H022457/1) to I.D.S, BIO2011-23446 to P.R., Swedish Governmental Agency for Innovation Systems (VINNOVA) and the Swedish Research Council (VR) to K.L. FWO Flanders (FWO, Belgium), Bilateral Research Cooperation with MOST (China) (2016YFE0109900) to W.X. and T.B. No competing interests declared.

\section{Author Contributions}

Conceptualization, B.O.-L., T.B., M.J.B., I.D.S., J.P.L., X.D.; Methodology, B.O.-L., E.C.M., T.L., A.B., X.D.; Software, X.D.; Formal analysis, B.O.-L., B.P., X.D.; Investigation, B.O.-L., E.C.M., S.K., C.S., W.X., A.L., O.N.; Resources, K.L., P.R., M.F., I.D., F.C., H.B., C.P., T.B., J.P.L., X.D.; Data Curation, B.P.; Writing - Original Draft, B.O.L., E.C.M., M.J.B., T.B., X.D.; Visualization, B.O.L., E.C.M.; Supervision, T.B., M.J.B., X.D.; Funding Acquisition, B.O.-L., T.B., M.J.B, H.B., F.C., C.P., X.D..

\section{Declaration of Interests}

The authors declare no competing interests.

\section{References}


1. Lynch, J.P. (2015). Root phenes that reduce the metabolic costs of soil exploration: opportunities for 21st century agriculture. Plant Cell and Environment 38, 1775-1784.

2. Lynch, J.P., Chimungu, J.G., and Brown, K.M. (2014). Root anatomical phenes associated with water acquisition from drying soil: targets for crop improvement. Journal of Experimental Botany 65, 6155-6166.

3. Robbins, N.E., and Dinneny, J.R. (2015). The divining root: moisture-driven responses of roots at the micro- and macro-scale. Journal of Experimental Botany 66, 2145-2154.

4. Morris, E.C., Griffiths, M., Golebiowska, A., Mairhofer, S., Burr-Hersey, J., Goh, T., von Wangenheim, D., Atkinson, B., Sturrock, C.J., Lynch, J.P., et al. (2017). Shaping 3D Root System Architecture. Current Biology 27, R919-R930.

5. Orman-Ligeza, B., Parizot, B., Gantet, P.P., Beeckman, T., Bennett, M.J., and Draye, X. (2013). Post-embryonic root organogenesis in cereals: branching out from model plants. Trends in Plant Science 18, 464-467.

6. Drew, M.C. (1975). COMPARISON OF EFFECTS OF A LOCALIZED SUPPLY OF PHOSPHATE, NITRATE, AMMONIUM AND POTASSIUM ON GROWTH OF SEMINAL ROOT SYSTEM, AND SHOOT, IN BARLEY. New Phytologist 75, 479-490.

7. Bao, Y., Aggarwal, P., Robbins, N.E., Sturrock, C.J., Thompson, M.C., Tan, H.Q., Tham, C., Duan, L.N., Rodriguez, P.L., Vernoux, T., et al. (2014). Plant roots use a patterning mechanism to position lateral root branches toward available water. Proceedings of the National Academy of Sciences of the United States of America 111, 9319-9324.

8. Yu, P., Gutjahr, C., Li, C.J., and Hochholdinger, F. (2016). Genetic Control of Lateral Root Formation in Cereals. Trends in Plant Science 21, 951-961.

9. Babe, A., Lavigne, T., Severin, J.P., Nagel, K.A., Walter, A., Chaumont, F., Batoko, H., Beeckman, T., and Draye, X. (2012). Repression of early lateral root initiation events by transient water deficit in barley and maize. Philosophical Transactions of the Royal Society B-Biological Sciences 367, 1534-1541.

10. Hong, J.H., Seah, S.W., and Xu, J. (2013). The root of ABA action in environmental stress response. Plant Cell Reports 32, 971-983.

11. De Rybel, B., Audenaert, D., Xuan, W., Overvoorde, P., Strader, L.C., Kepinski, S., Hoye, R., Brisbois, R., Parizot, B., Vanneste, S., et al. (2012). A role for the root cap in root branching revealed by the non-auxin probe naxillin. Nature Chemical Biology 8, 798-805.

12. De Smet, I., Vassileva, V., De Rybel, B., Levesque, M.P., Grunewald, W., Van Damme, D., Van Noorden, G., Naudts, M., Van Isterdael, G., De Clercq, R., et al. (2008). Receptor-like kinase ACR4 restricts formative cell divisions in the Arabidopsis root. Science 322, 594-597.

13. Vanneste, S., De Rybel, B., Beemster, G.T., Ljung, K., De Smet, I., Van Isterdael, G., Naudts, M., lida, R., Gruissem, W., Tasaka, M., et al. (2005). Cell cycle progression in the pericycle is not sufficient for SOLITARY ROOT/IAA14mediated lateral root initiation in Arabidopsis thaliana. Plant Cell 17, 30353050.

14. Xuan, W., Audenaert, D., Parizot, B., Moller, B.K., Njo, M.F., De Rybel, B., De Rop, G., Van Isterdael, G., Mahonen, A.P., Vanneste, S., et al. (2015). Root Cap- 
Derived Auxin Pre-patterns the Longitudinal Axis of the Arabidopsis Root. Current Biology 25, 1381-1388.

15. Hubick, K.T., Taylor, J.S., and Reid, D.M. (1986). The effect of drought on levels of abscisic acid, cytokinins, gibberellins and ethylene in aeroponically-grown sunflower plants. Plant Growth Regulation 4, 139-151.

16. Gonzalez-Guzman, M., Pizzio, G.A., Antoni, R., Vera-Sirera, F., Merilo, E., Bassel, G.W., Fernandez, M.A., Holdsworth, M.J., Perez-Amador, M.A., Kollist, H., et al. (2012). Arabidopsis PYR/PYL/RCAR receptors play a major role in quantitative regulation of stomatal aperture and transcriptional response to abscisic acid. The Plant cell 24, 2483-2496.

17. Dubrovsky, J.G., Gambetta, G.A., Hernandez-Barrera, A., Shishkova, S., and Gonzalez, I. (2006). Lateral root initiation in Arabidopsis: developmental window, spatial patterning, density and predictability. Annals of botany 97, 903-915.

18. De Smet, I., Tetsumura, T., De Rybel, B., Frey, N.F., Laplaze, L., Casimiro, I., Swarup, R., Naudts, M., Vanneste, S., Audenaert, D., et al. (2007). Auxindependent regulation of lateral root positioning in the basal meristem of Arabidopsis. Development 134, 681-690.

19. De Rybel, B., Vassileva, V., Parizot, B., Demeulenaere, M., Grunewald, W., Audenaert, D., Van Campenhout, J., Overvoorde, P., Jansen, L., Vanneste, S., et al. (2010). A novel aux/IAA28 signaling cascade activates GATA23-dependent specification of lateral root founder cell identity. Current biology : CB 20, $1697-$ 1706.

20. De Smet, I. (2011). Lateral root initiation: one step at a time. New Phytologist 193, 867-873.

21. Moreno-Risueno, M.A., Van Norman, J.M., Moreno, A., Zhang, J., Ahnert, S.E., and Benfey, P.N. (2010). Oscillating gene expression determines competence for periodic Arabidopsis root branching. Science 329, 1306-1311.

22. Van Norman, J.M., Zhang, J., Cazzonelli, C.I., Pogson, B.J., Harrison, P.J., Bugg, T.D., Chan, K.X., Thompson, A.J., and Benfey, P.N. (2014). Periodic root branching in Arabidopsis requires synthesis of an uncharacterized carotenoid derivative. Proceedings of the National Academy of Sciences of the United States of America 111, E1300-1309.

23. Lavenus, J., Goh, T., Roberts, I., Guyomarc'h, S., Lucas, M., De Smet, I., Fukaki, H., Beeckman, T., Bennett, M., and Laplaze, L. (2013). Lateral root development in Arabidopsis: fifty shades of auxin. Trends in plant science 18, 450-458.

24. Benkova, E., Michniewicz, M., Sauer, M., Teichmann, T., Seifertova, D., Jurgens, G., and Friml, J. (2003). Local, efflux-dependent auxin gradients as a common module for plant organ formation. Cell 115, 591-602.

25. Himanen, K., Boucheron, E., Vanneste, S., de Almeida Engler, J., Inze, D., and Beeckman, T. (2002). Auxin-mediated cell cycle activation during early lateral root initiation. The Plant cell 14, 2339-2351.

26. Ludwig-Muller, J. (2011). Auxin conjugates: their role for plant development and in the evolution of land plants. Journal of experimental botany 62, 17571773. 
27. Novak, O., Henykova, E., Sairanen, I., Kowalczyk, M., Pospisil, T., and Ljung, K. (2012). Tissue-specific profiling of the Arabidopsis thaliana auxin metabolome. The Plant journal : for cell and molecular biology 72, 523-536.

28. De Diego, N., Rodriguez, J.L., Dodd, I.C., Perez-Alfocea, F., Moncalean, P., and Lacuesta, M. (2013). Immunolocalization of IAA and ABA in roots and needles of radiata pine (Pinus radiata) during drought and rewatering. Tree physiology 33, 537-549.

29. De Smet, I., Signora, L., Beeckman, T., Inze, D., Foyer, C.H., and Zhang, H. (2003). An abscisic acid-sensitive checkpoint in lateral root development of Arabidopsis. The Plant journal : for cell and molecular biology 33, 543-555.

30. Xiong, L., Wang, R.G., Mao, G., and Koczan, J.M. (2006). Identification of drought tolerance determinants by genetic analysis of root response to drought stress and abscisic Acid. Plant physiology 142, 1065-1074.

31. Zhao, Y., Xing, L., Wang, X., Hou, Y.J., Gao, J., Wang, P., Duan, C.G., Zhu, X., and Zhu, J.K. (2014). The ABA receptor PYL8 promotes lateral root growth by enhancing MYB77-dependent transcription of auxin-responsive genes. Science signaling 7 , ra53.

32. Zhang, H., Han, W., De Smet, I., Talboys, P., Loya, R., Hassan, A., Rong, H., Jurgens, G., Paul Knox, J., and Wang, M.H. (2010). ABA promotes quiescence of the quiescent centre and suppresses stem cell differentiation in the Arabidopsis primary root meristem. The Plant journal : for cell and molecular biology 64, 764-774.

33. Moriwaki, T., Miyazawa, Y., Fujii, N., and Takahashi, H. (2012). Light and abscisic acid signalling are integrated by MIZ1 gene expression and regulate hydrotropic response in roots of Arabidopsis thaliana. Plant, cell \& environment 35, 1359-1368.

34. Bahrun, A., Jensen, C.R., Asch, F., and Mogensen, V.O. (2002). Drought-induced changes in xylem $\mathrm{pH}$, ionic composition, and $A B A$ concentration act as early signals in field-grown maize (Zea mays L.). Journal of experimental botany 53 , 251-263.

35. Irizarry, R.A., Hobbs, B., Collin, F., Beazer-Barclay, Y.D., Antonellis, K.J., Scherf, U., and Speed, T.P. (2003). Exploration, normalization, and summaries of high density oligonucleotide array probe level data. Biostatistics 4, 249-264.

36. Gautier, L., Cope, L., Bolstad, B.M., and Irizarry, R.A. (2004). affy--analysis of Affymetrix GeneChip data at the probe level. Bioinformatics 20, 307-315.

37. Smyth, G.K. (2004). Linear models and empirical bayes methods for assessing differential expression in microarray experiments. Statistical Applications in Genetics and Molecular Biology 3, Article 3.

38. Smyth, G.K., Michaud, J., and Scott, H.S. (2005). Use of within-array replicate spots for assessing differential expression in microarray experiments. Bioinformatics 21, 2067-2075.

39. Benjamini, Y., and Hochberg, Y. (1995). CONTROLLING THE FALSE DISCOVERY RATE - A PRACTICAL AND POWERFUL APPROACH TO MULTIPLE TESTING. J. R. Stat. Soc. Ser. B-Methodol. 57, 289-300.

40. Van Bel, M., Diels, T., Vancaester, E., Kreft, L., Botzki, A., Van de Peer, Y., Coppens, F., and Vandepoele, K. (2018). PLAZA 4.0: an integrative resource for 
functional, evolutionary and comparative plant genomics. Nucleic acids research 46, D1190-D1196.

41. Lobet, G., Pages, L., and Draye, X. (2011). A Novel Image-Analysis Toolbox Enabling Quantitative Analysis of Root System Architecture. Plant Physiology 157, 29-39.

42. Xuan, W., Opdenacker, D., Vanneste, S., and Beeckman, T. (2018). Long-Term In Vivo Imaging of Luciferase-Based Reporter Gene Expression in Arabidopsis Roots. In Root Development: Methods and Protocols, D. Ristova and E. Barbez, eds. (New York, NY: Springer New York), pp. 177-190.

43. Xuan, W., Band, L.R., Kumpf, R.P., Van Damme, D., Parizot, B., De Rop, G., Opdenacker, D., Moeller, B.K., Skorzinski, N., Njo, M.F., et al. (2016). Cyclic programmed cell death stimulates hormone signaling and root development in Arabidopsis. Science 351, 384-387.

\section{Main Text Figure Legends}

Figure 1. Xerobranching response is observed in maize and barley grown in soil.

$(A$ and B) X-ray CT images of 16 day old maize (B73) $(n=5)(A)$ and barley (B83) $(n=3)(B)$ plants growing through a $2 \mathrm{~cm}$ air gap. Root tissue is falsecolored in white and soil is false-colored in brown. (C-H) Representative confocal images of cross sections taken from maize (C-E) and barley (F-H) primary roots in the top section of the soil ( $C$ and $F$ ), air gap ( $D$ and $G$ ) and bottom section of soil $(E$ and $H)$. Sample size of 54 images from 3 maize plants and 47 images from 3 barley plants. Scale bar $=0.1 \mathrm{~mm}$. See Figure S1 for the response to submergence and laser ablation tomography images. See Video $\mathrm{S} 1$ for a movie of xerobranching in maize.

Figure 2. Root transcriptome changes during xerobranching in barley involves hormonal response pathways, such as ABA which can mimic xerobranching when applied transiently.

(A) Hierarchical clustering of the 755 differentially expressed genes in barley (cv Derkado) ( $p$-value $\leq 0.01$, fold-change $\geq 1.5$ ) showing two independent biological replicates per condition. Low expression levels in blue, high expression levels in yellow. (B) Gene ontology term enrichment for plant hormones in the list of differentially expressed genes in barley. GO identifiers are enclosed in brackets. The number of differentially expressed genes experimentally associated with each term is enclosed within brackets. Bars 
indicate the $\log _{2}$ (Enrichment) for each term. Categories related with $A B A$ highlighted in yellow and auxin in blue. (C) Quantification of ABA content in barley root tissue subjected to a 4 hour long transient WD. Bars are means \pm $\mathrm{SD}$ for $\mathrm{n}=5$ root complete root systems. Asterisks indicate statistically significant differences between treated and control plants (Student's $t$ test; $p<$ 0.01). ( $D$ and $E$ ) Length of the repression zone (RZ) and branching pattern in barley (B83) and maize (B73) root tissue after a 6 hour long treatment with 50 $\mu M$ ABA in LR repression experiments in aeroponics. Bars $=0.5 \mathrm{~cm}$. See Data S1 for the list of transcripts differentially expressed upon water deficit. See Figure S2 for qRT-PCR validation of the water deficit microarray. See Figure S3 for confirmation of the formation of a repression zone in agar plates.

Figure 3. PYR/PYL/RCAR dependent signalling is required for transient ABA stimulated LR repression in Arabidopsis.

(A) Experimental design for Arabidopis root system exposed to DMSO (Control) or $A B A(+A B A)$ for 2 days in LR repression experiments. (B) Length of the repression zone (RZ) in Arabidopsis seedlings grown as described in A for 2 days with various ABA concentrations $(n=12)$. (C) Average Arabidopsis root growth rate during 2 days of treatment with given ABA concentrations $(n=12)$. (D) Average d1 offset between the position of the root tip at the beginning of ABA treatment and the last emerged $L R(n=12)$. (E) Average $d 2$ offset between the position of the root tip at the end of ABA treatment and the first emerged LR $(n=12)$. Bars are means \pm SD. Different letters indicate significant differences with $p<0.001$ according to Tukey's HSD test after ANOVA. (F) Branching pattern of $12 \mathrm{dpg}$ Arabidopis root systems of Col-0 and ABA-response mutants as indicated, exposed to DMSO (- ABA) or $30 \mu \mathrm{M}$ ABA (+ ABA) for 2 days. White lines demark the root segments that grew during 2 days of transient DMSO or ABA treatment. ( $G$ and $H$ ) LR number in the root segments that grew in the presence of DMSO or ABA in 2 days. Note this is not equivalent to the $R Z$, due to $d 1$ and $d 2$, and therefore a few LRs are present in the ABA treatment as these are formed in d2. Data are represented as percentage $(n=31$ and $n$ $=36$ respectively).

Figure 4. Transient ABA treatment alters DR5 oscillations in the root oscillation zone in Arabidopsis and affects auxin accumulation in maize.

(A) Representative DR5:Luciferase luminescence in the LR repression experiments viewed by using time-lapse imaging. 4 or $5 \mathrm{dpg}$ Arabidopsis seedlings grown on standard media were transferred to standard media with DMSO (- ABA) or $30 \mu \mathrm{M}$ ABA (+ABA) and imaged every 10 minutes up to 18 hours in a dark imaging chamber $(n=10)$. Black-filled arrowheads indicate the position of prebranch sites that appear during the time-lapse imaging and subsequent formation of DR5 maxima that indicates the position of future LR primordia. White line indicates the position of the oscilation zone (OZ). White 
stars mark the position where DR5 oscillation pulses should be present by virtue of their position along root. (B) Branching pattern of barley roots in auxin complementation assays. Plants grown in aeroponics were exposed for 1 hour to nutrient solution (Control, $n=14)$ or to $75 \mu M$ of 2,4-D $(n=15)$, IAA $(n=19)$ or NAA $(n=18)$ in nutrient solution, then treated for 6 hours with $50 \mu \mathrm{M}$ ABA and to $50 \mu M$ ABA alone without any pre-treatment $(n=17)$. Roots were imaged after 7 days of growth and the average number of LRs within the repression zone (RZ) was calculated (C), and compared to control conditions (Control) when no pre-treatment was applied.. Bars are means $\pm \mathrm{Cl}$. Different letters indicate significant differences with $p<0.05$ according to Tukey's HSD test after ANOVA. (D, E and F) Changes in levels of given auxin metabolites in maize in four successive $0.5 \mathrm{~cm}$ long root segments starting from the root tip when treated with $A B A(n=3)$. Presented as a percent of non treated roots. (D) Levels of free IAA upon 2 hour, 4 hour and 6 hour long $50 \mu \mathrm{M}$ ABA treatments. (E) Levels of IAA precursors upon 2 hour long $50 \mu \mathrm{M}$ ABA treatment: Tryptophan (Trp), Tryptamine (Tra), Indole-3-pyruvic acid (IpyA). (F) Levels of IAA conjugates upon 2 hour long $50 \mu \mathrm{M}$ ABA treatment: oxindole-3-acetic acid (oxIAA), indole-3-acetyl-aspartate (IAA-Asp), and indole-3-acetyl-glutamate (IAA-Glu). Bars are means \pm SD. Asterisks indicate statistically significant differences between treated and control plants (Student's $t$ test; * and ** correspond to $P$ value of $0.05>p>0.01$ and $0.01>p>0.001$, respectively). (G) Schematic representation of the xerobranching response. When roots are not in contact with water as they enter a soil macropore, pre-branch site formation is repressed by ABA response pathways causing immediate repression of pre-branch site formation and hence, LR development. See Data S2 for the dose response of auxin pre-treatments in the transient ABA assay. See Figure S4 for decreased auxin response in Arabidopsis, decreases in DR5 maxima in maize and auxin treatments post transient ABA application in barley. See Video S2 for movies of the images presented in Figures 4A and S4A.

\section{STAR Methods}

\section{CONTACT FOR REAGENT AND RESOURCE SHARING}

Further information and requests for resources and reagents should be directed to and will be fulfilled by the Lead Contact, Xavier Draye xavier.draye@uclouvain.be.

\section{EXPERIMENTAL MODEL AND SUBJECT DETAILS}

In this report we use Arabidopsis thaliana Col-0, Zea mays (B73 and Emmerson) and Hordeum vulgare (cv Derkado and B83). 


\section{Arabidopsis thaliana}

In all experiments with Arabidopsis, seeds were sterilized with chlorine gas and stratified at $4^{\circ} \mathrm{C}$ for 2 days in water. After cold treatment, seeds were sown over solid half-strength MS growth medium (per litre: $2.15 \mathrm{~g}$ MS salts, $0.1 \mathrm{~g}$ myo-inositol, $0.5 \mathrm{~g}$ MES, $10 \mathrm{~g}$ sucrose, $8 \mathrm{~g}$ plant tissue culture agar; $\mathrm{pH}$ $=5.7$ with $\mathrm{KOH})$ and grown vertically under continuous light $\left(110 \mu \mathrm{E} \mathrm{m}^{-2} \mathrm{~s}^{-1}\right.$ photosynthetically active radiation, supplied by cool-white fluorescent tungsten tubes, Osram) for 4 - 5 days.

\section{Zea mays (B73 and Emmerson) and Hordeum vulgare (cv Derkado and} B83)

Barley and maize seeds were left to germinate on a vertical filter paper for 72 hours in the dark at $18^{\circ} \mathrm{C}$ and $22^{\circ} \mathrm{C}$, respectively (unless otherwise stated). The root system was reduced to a single embryonic root, preferably the most vertical one, unless grown for soil columns. Germinated seeds were transferred to $40 \times 60 \times 60 \mathrm{~cm}$ aeroponic systems containing $5 \mathrm{~L}$ of nutrient solution sprayed for 15 s every 5 min. Barley seedlings were grown in a halfstrength Hewitt solution (2 mM Ca(NO3)2 - 4H2O, 2 mM KNO3, 0.75 mM MgSO4 · 7H2O, 0.67 mM NaH2PO4 - 2H2O, 0.05 mM FeEDTANa, $0.03 \mu \mathrm{M}$ (NH4)6Mo7O24 - 4H2O, $50 \mu \mathrm{M} \mathrm{NaCl}, 25 \mu \mathrm{M} \mathrm{H3BO3,} 5 \mu \mathrm{M} \mathrm{MnCl}$ - 4H2O, $0.5 \mu \mathrm{M}$ CuSO $\cdot 5 \mathrm{H} 2 \mathrm{O}, 0.5 \mu \mathrm{M} \mathrm{ZnSO} 4 \cdot 7 \mathrm{H} 2 \mathrm{O}, 0.6 \mathrm{mM} \mathrm{Na} 2 \mathrm{SiO} 3 \cdot 5 \mathrm{H} 2 \mathrm{O} ; \mathrm{pH}$ 5.8). Maize seedlings were grown in a modified Hoagland solution ( $2 \mathrm{mM}$ KNO3, 2 mM Ca(NO3)2 · 4H2O, 2 mM MgSO4 · 7H2O, 1 mM NH4NO3, 1 $\mathrm{mM} \mathrm{KH} 2 \mathrm{PO} 4,10 \mu \mathrm{M} \mathrm{MnCl} 2 \cdot 4 \mathrm{H} 2 \mathrm{O}, 50 \mu \mathrm{M}$ CuSO4, $1 \mu \mathrm{M} \mathrm{ZnSO} 4 \cdot 7 \mathrm{H} 2 \mathrm{O}, 0.2$ $\mu \mathrm{M}$ H3Mo4; $\mathrm{pH}$ 5.0). All experiments were conducted in a PGV36 growth chamber (Conviron, Winnipeg, Canada) with a day : night temperature regime of $18^{\circ} \mathrm{C}: 16^{\circ} \mathrm{C}$ for barley and $22^{\circ} \mathrm{C}: 20^{\circ} \mathrm{C}$ for maize, a relative humidity of 70 per cent, a $16 \mathrm{~h}$ long photoperiod and a photosynthetically active radiation of $350 \mu \mathrm{mol} m-2 \mathrm{~s}-1$.

\section{METHOD DETAILS}

\section{CT scanning experiments}


Barley (B83) and maize (B73 and Emmerson) plants were grown in columns 10 $\mathrm{cm}$ tall and $3.5 \mathrm{~cm}$ wide filled with sandy loam. Seeds were soaked for 30 minutes and then stratified at $4^{\circ} \mathrm{C}$ for 3 days on wet filter paper. Seeds were germinated for 1 day on filter paper at room temperature before being transferred to soil. A $2 \mathrm{~cm}$ air gap was created in the middle of the column using a mesh insert to hold the top layer of soil up while allowing the root to pass through. Plants were grown for 16 days at $20^{\circ} \mathrm{C}$ in 16 hour light conditions and watered from the top and the bottom every other day before imaging with $\mathrm{X}$-ray $\mu \mathrm{CT}$ scanning. 3 barley, 5 B73 maize and 3 Emmerson maize plants have been imaged with this experimental set up and all show LR repression.

In order to test root growth through water filled gaps the whole column was submerged under water. A water aerator was used to prevent hypoxia in the roots. The columns were removed from water 24 hours before CT scanning in order to dry the soil.

$\mathrm{X}$-ray $\mu \mathrm{CT}$ scanning using a phoenix v|tome|x m industrial scanner (GE Sensing and Inspection Technologies, Wunstorf, Germany), at $160 \mathrm{kV}$ and 160 $\mu \mathrm{A}$. The distance between the $\mathrm{X}$-ray source and the sample and the $\mathrm{X}$-ray source and the detector was $122.805 \mathrm{~mm}$ and $818.69 \mathrm{~mm}$, respectively, resulting in a magnification of $\times 6.666$ and a spatial resolution of $30 \mu \mathrm{m}$. Each scan acquired 2160 projection images over a $360^{\circ}$ rotation of the sample using a detector exposure time of $250 \mathrm{~ms}$ in 'fast mode' resulting in a total scan time of 9 minutes. Data was reconstructed using datos|x software (GE Sensing) and visualised using VGStudio MAX V.2.2 (Volume Graphics, Heidelberg, Germany). Images show a $4.8 \mathrm{~cm}$ long section of the column.

\section{Imaging cereal cross sections}

Plants were grown as described above and sectioned to view root cross sections. In the first experimental procedure roots were extracted from soil, solidified in agar and then sectioned using a vibratome. The slices were stained with Fluorescent Brightener 28 and then imaged with a confocal microscope. In the second experimental procedure roots were extracted from soil and preserved in $75 \%$ ethanol. They were then imaged using laser ablation 
tomography (LAT) where the root was simultaneously cut and imaged at $1 \mathrm{~mm}$ intervals.

\section{Expression studies in barley}

The sampling strategy was designed to monitor gene expression at $-4,-2,0$ and 2 hours relative to the first asymetric division, in conditions that enable (A) or repress (B) LR formation. $5 \mathrm{~mm}$ long segments were sampled such that the first sampling (-4h position) targets a region distal to the first asymetric divisions, and the last sampling ( $+2 \mathrm{~h}$ position) targets a region proximal to the first asymetric division.

From previous work, we considered that the first asymmetric cell division occurs on average $12 \mathrm{~mm}$ proximal from the root tip, while the next divisions occur at $20 \mathrm{~mm}$ from the tip. Based on this, a sample length of $5 \mathrm{~mm}$ was chosen, as a compromise between maximising the amount of RNA per sample and minimising the probablity to capture cells undergoing the second round of divisions. As a no-LR control, we relied on a described LR repression system [9]. In this system, the first asymetric divisions are repressed within 2 hours of a water deficit treatment. The treatment can be applied as long as 8 hours. In the conditions of these experiments, barley roots grow at ca. $1 \mathrm{~mm} \cdot \mathrm{hr}^{-1}$. During an 8 hour-long treatment, a $8 \mathrm{~mm}$ long root segment is formed where LR formation has been repressed.

The following indicates the likely number of formative divisions in each sample, assuming a LR density of $1 \mathrm{LR} \cdot \mathrm{mm}^{-1}$. At the $-4 \mathrm{~h}$ position (T0), the samples in $A$ and $B$ conditions do not contain any first asymetric divisions. At the $-2 h$ position (T2), A samples are likely to contain one LR initiation engaged in asymetric divisions, against zero in the $B$ samples where these sites have been repressed. At the -Oh position (T4), A samples are likely to contain two-three LR initiation engaged in asymetric divisions, against zero in the $B$ samples where these sites have been repressed. At the $+2 h$ position (T6), A samples are likely to contain four LR initiations engaged in asymetric divisions, against zero in the B samples where these sites have been repressed. 
Preliminary tests indicated that a $6 \mathrm{~cm}$ long fragment would give the required amount of RNA for one chip hybridization. We therefore collected 12 independent biological samples for each chip. The whole procedure was repeated two times, amounting to a total of 24 independent biological samples (two chips) per condition and time point.

RNA was isolated from frozen root segments according to the SV Total RNA Isolation Systems (Promega). After DNAse treatment (Promega), RNA samples were quantified by using Nanodrop spectrophotometer (Nanodrop Technologies) and then confirmed by RNA electrophorese. $5.3 \mu \mathrm{g}$ of total RNA was used for cDNA synthesis, labelling and fragmentation according to One Cycle Target Labelling kit (Affymetrix), as for manufacture protocol. The samples were hybridized to the arrays for 16 hours at $45^{\circ} \mathrm{C}$, washed on GeneChip Fluids Station (Affymetrix) and imaged on GeneChip Scanner (Afftmetrix). The resulting data were processed for quality check in MAS 5.0 (Affymetrix). The expression values have been normalized using the robust multi-array average method [35]. Differential analysis was performed using linear models and empirical Bayes methods within affy and limma R packages [www.r-project.org, 36, 37, 38]. Raw p-values were adjusted using the Benjamini-Hochberg method to control the FDR [39]. Affymetrix probesets annotation was retrieved from Affymetrix website (Affymetrix_Barley1.na34.annot, http://www.affymetrix.com/) and genes that are controls of the microarray were discarded. Two-factor ANOVA p-values were computed using MeV 4.9 (mev.tm4.org). The data discussed in this publication have been deposited in NCBI's Gene Expression Omnibus and are accessible through GEO Series accession number GSE67367 (http://www.ncbi.nlm.nih.gov/geo/query/acc.cgi?acc=GSE67367). Genes have been selected if they could satisfy the following criteria: significant regulation upon drought treatment in at least one of three pairwise comparisons (Drought T2 VS T0, Drought T4 VS Drought T2, Drought T6 VS Drought T4) and dependant on the treatment, the time and the interaction in comparison to control time points without drought treatment (FC $\geq 1.5$, FDR corrected pvalue $\leq 0.01$, two factor Anova pValue $\leq 0.01$ ). The homologous equivalence between probesets contigs and Arabidopsis AGI gene models was realized using the 
genelist suite on PLEXdb (http://www.plexdb.org/modules/glSuite/) with default parameters.

The heatmap of the hierarchical clustering was performed on the list of 755 differentially expressed genes described previously using MeV 4.9 (mev.tm4.org) with an average pearson correlation linkage.

The gene ontology enrichment was performed on Plaza 4.0 [40] using a set of 461 Arabidopsis orthologs deriving from the list of 755 differentially expressed genes described previously. The results (all significant with a pvalue $\leq 0.01$ ) were parsed to retain only categories related with the major plant hormones (abscisic acid, auxins, brassinosteroids, cytokinins, ethylene, gibberellins and jasmonic acid).

The microarray validation was performed by qRT-PCR (see Figure S2). RNA was isolated using FastRNA Pro Green kit (MP Biomedicals). OD 260/280 ratios were determined using a Nanodrop ND-1000 (Nanodrop Technologies Inc.). $1 \mu \mathrm{g}$ of the total RNA was reverse transcribed using the Superscript III reverse transcriptase (invitrogen) according to the protocol. cDNA samples were diluted 2 times with sterile milliQ water. For each qPCR, $1 \mu l$ of $2 x$ diluted cDNA sample, $20 \mu \mathrm{l}$ of $200 \mathrm{nM}$ of gene-specific primers and $10 \mu \mathrm{l}$ of ABsolute Blue SYBR green Fluor (Thermo Scientific) mix was added. The RT-qPCRs were run on a IcyclerlQ (Biorad). The qPCR program run consisted of a first step at $95^{\circ} \mathrm{C}$ for $15 \mathrm{~min}$ and afterwards 40 cycles alternating between $15 \mathrm{~s}$ at $95^{\circ} \mathrm{C}, 60 \mathrm{~s}$ at $60^{\circ} \mathrm{C}$ and $30 \mathrm{~s} 72^{\circ} \mathrm{C}$. The expression of Contig_10961 relative to control gene expression EF1a (barley Elongation Factor 1-alpha, gi : z50789) was estimated by using the $\Delta \Delta C T$ method $(n=2)$. This choice of Contig_10961 was motivated by the fact that 1) expression of this gene is downregulated rapidly upon WD treatment, and 2) bZIP transcription factors are known to regulate developmental responses to stress.

\section{Arabidopsis datasets compendium analysis}

Previously published datasets [11-14] were retrieved from the following respective Gene Expression Omnibus (http://www.ncbi.nlm.nih.gov/geo/) under the accession numbers GEO: GDS1515, GSE6349, GSE42896 and 
GSE59426 and were independently analyzed using the same procedure as previously described for the barley microarray. For the enrichment (ratio between the expected and observed number of genes differentially expressed in the barley and a given Arabidopsis dataset), $p$-values were generated using the Fisherexact Excel addin (http://www.obertfamily.com/software/fisherexact.html).

Genes were considered to be significantly regulated in each independent experiment if they could satisfy the following respective conditions: (i) absolute fold change $F C \geq 1,5$, adjusted $p$-value $\leq 0.01$, between 0 and 6 hours upon lateral root inducible system in the control plants, and a TWO-WAY ANOVA pvalue $\leq 0.01$ for the interaction of the treatment and the genotype (s/r), (ii) absolute $F C \geq 2$, adjusted $p$-value $\leq 0.01$ between 0 and 6 hours upon lateral root inducible system in the sorted pericycle cells, (iii) absolute $F C \geq 2$, adjusted p-value $\leq 0.01$ between 0 and 2 hours upon treatment with both compound (NAA and naxillin) during the time course upon lateral root induction system, (iv) absolute fold change $F C \geq 1,5$, adjusted $p$-value $\leq 0.01$, between 0 and 6 hours upon IBA treatment in the control plants, and a TWO-WAY ANOVA p-value $\leq 0.01$ for the interaction of the treatment and the genotype (ibr1 ibr3 ibr10).

\section{Aeroponic growth system}

In aeroponics experiments, 40 seedlings (4 dpg (barley) or $3 \mathrm{dpg}$ (maize)) were grown with $5 \mathrm{~L}$ of nutrient solution $(\mathrm{pH}=5.8)$. For transient water deficit experiments the plants were grown for 12 days when half of the seeds were subjected to a transient WD treatment. The treatment was applied by interrupting the nutrient supply for 4 hours during the night phase. To apply a transient $\mathrm{ABA}$ treatment, after one or two days, the nutrient solution was replaced during up to 8 hours with a fresh nutrient solution with or without 50 $\mu \mathrm{M}$ ABA (stock: 50 mM ABA (Alfa Aesar) diluted in dimethyl sulfoxide (DMSO, Sigma-Aldrich). Root growth rates and lengths of repression zones were estimated as as follows. Greyscale images of seedlings were captured using a modified 600 dots per inch flatbed transparency scanner (Medion, Germany). They were obtained at the onset, at the end of the ABA treatment and after 7 days. Images were analysed using SmartRoot and SAS software for 
computation of $\mathrm{RZ}$ length and for data manipulation preceding statistical analysis [41].

\section{Quantification of ABA content}

Barley seedlings were grown in aeroponics as described above. Each sample type represents five complete root systems. The peak corresponding to $A B A$ concentration was quantified by ELISA with an anti-ABA antibody MAC 252.

\section{Arabidopsis agar plate growth assays}

Scans of the plates were taken with V700 (Epson). Figures were arranged in Photoshop CS3 without modifications. For transient ABA treatments, $5 \mathrm{dpg}$ seedlings were transferred on square Petri plates with or without increasing concentrations of ABA and the exact positions of root tips were marked. After $2 \mathrm{~d}$, plates were scanned and seedlings were transferred on new plates without ABA in the same order. After $7 \mathrm{~d}$, positions of emerged LRs were marked under binocular (S6D, Leica) and plates were scanned again. For microscopic analysis of repression zone, root segments corresponding to repression zones were cleaned overnight in chloral hydrate solution and observed with BX53 microscope (Olympus) equipped with DS-Fi1 (Nicon) camera. Each data point represents a mean from at least 10 seedlings. Images were analyzed with Image J software to estimate the length of repression zones and the longitudinal position of individual LR flanking repression zones preceding statistical analysis.

\section{In vivo imaging for DR5:Luciferase}

For DR5:Luciferase imaging, 4 or 5 dpg Arabidopsis seedlings were transferred on plates with DMSO or $30 \mu \mathrm{M}$ ABA. Before transfer, the plates were sprayed with $400 \mu \mathrm{l}$ of $1 \mathrm{mM}$ D-luciferine (Duchefa Biochemie) using a pump spray and left in darkness to dry. The DR5:Luciferase signal was recorded by a Lumazone machine carrying a charge-coupled device (CCD) camera (Princeton Instruments, Trenton, NJ, USA) [42]. The CCD camera that is controlled by a WinView/32 software took movies of the DR5:Luciferase [21] expression automatically every 10 minutes (exposure time, 10 minutes) for up to 24 hours. The picture series were saved as TIFF format for further analysis. 


\section{In vivo imaging for DR5rev:3xVENUS-N7}

For DR5rev:3xVENUS-N7 imaging experiments, 4 or $5 \mathrm{dpg}$ seedlings were transferred directly on plates containing DMSO or $30 \mu \mathrm{M}$ ABA. Photographs were taken every 2 minutes (exposure time 1 second) up to 24 hours for DR5rev:3xVENUS-N7 line by using a 90 degree tilted stereo microscope (Olympus MVX10), which enables visualizing fluorescence signal in a vertical growing root [43]. The picture series were saved as TIFF format for further analysis.

\section{Repression zone formation in cereals on agar plates}

In $A B A$ experiments on agar square plates, $3 \mathrm{dpg}$ barley seedlings were transferred from filter paper (Waterman) to the square Petri plates with $50 \mathrm{ml}$ of nutrient solution $(\mathrm{pH}=5.8)$ supplemented with $1.5 \%$ of agarose alone or with ABA to the final concentration of $50 \mu \mathrm{M}$ (repression zone experiment) with 10 seedlings per each plate. The same amount of DMSO was added to the control solution. Positions of root tips were marked. In repression zone formation assay, seedlings were grown vertically for $6 \mathrm{~h}$, scanned and then transferred to the aeroponics with nutrient solution. After 7 days in aeroponics, seedlings were scanned again and images were processed with Image J software. Mean values were determined from two biological replicates.

\section{Microscopy analysis in cereals}

For DR5rev:RFP experiments in maize, apical root segments were fixed in 4 per cent para-formaldehyde in phosphate buffer for 1 hour at $4^{\circ} \mathrm{C}$, mounted with distilled water and photographed with a Leica epi-fluorescence binocular with a filter set for rhodamine. Images were taken with an AxioCam (Zeiss).

\section{Auxin pretreatment assays}

In auxin pretreatment assays, seedlings were exposed to auxin for 1 hour before the start of the ABA treatment using a nutrient solution $(\mathrm{pH}=5.8)$ supplemented with increasing concentrations $(0,1,10,50,75$ and $100 \mu \mathrm{M})$ of different auxins: $\alpha-N a p h t h a l e n e$ acetic acid (NAA, Sigma-Aldrich), indole-3acetic acid (IAA, Sigma-Aldrich) and 2,4-Dichlorophenoxyacetic acid (2.4D, 
Sigma-Aldrich) and washed briefly before the ABA treatment. See Data S2 for initial evaluation. Only results for $75 \mu \mathrm{M}$ are shown on Figure 4.

\section{Quantification of IAA metabolites}

$3 \mathrm{dpg}$ maize seedlings were transferred to aeroponic box containing nutrient solution. After one day, half of the seeds were subjected to ABA treatment, where $50 \mu \mathrm{M}$ ABA was added to nutrient solution for varying lengths of time. Small fragments of roots, $0.5 \mathrm{~cm}$ each up to $2 \mathrm{~cm}$ from the root tip from at least three roots per sample were harvested and immediately frozen in liquid nitrogen. IAA metabolites were quantified from $20 \mathrm{mg}$ of frozen roots using LC-MRM-MS (liquid chromatography/multiple reaction monitoring/mass spectrometry) [27]. Three biological replicates were performed per sample type.

\section{QUANTIFICATION AND STATISTICAL ANALYSIS}

All data analyses were performed with $R$ software package, v. 2.15, with different letters indicating significant differences according to Tukey's HSD test after ANOVA, unless stated otherwise. Statistical details can be found in figure legends.

\section{DATA AND SOFTWARE AVAILABILITY}

\section{Microarray data}

The microarray data discussed in this publication have been deposited in NCBI's Gene Expression Omnibus and are accessible through GEO Series accession number GSE67367 (http://www.ncbi.nlm.nih.gov/geo/query/acc.cgi?acc=GSE67367).

\section{Accession Numbers}

Sequence data from the genes investigated can be found in the GenBank/European Molecular Biology Laboratory (EMBL) data libraries under accession numbers: At4g17870 (PYR1), At5g46790 (PYL1), At2g26040 (PYL2), At1g73000 (PYL3), At2g38310 (PYL4), At5g05440 (PYL5), At2g40330 (PYL6), At4g01026 (PYL7), At5g53160 (PYL8), At1g01360 (PYL9). The Arabidopsis sextuple mutant of pyr1 pyl1 pyl2 pyl4 pyl5 pyl8 (pyr/pyl 112458) 
was previously described [16] and crossed with pyl9 (SALK_08361) in order to obtain the heptuple pyr/pyl 1124589 mutant.

\section{KEY RESOURCES TABLE}

Separate file uploaded.

\section{Supplemental Items Figure Legends}

Data S1. The list of transcripts differentially expressed upon water deficit, related to Figure 2.

Data S2. Initial evaluation of the dose response to auxin pre-treatments in transient ABA assay, related to Figure 4.

Video S1. Video of maize xerobranching, related to Figure 1.

A video showing the CT reconstruction of a maize root growing through a soil macropore and displaying xerobranching. The video runs through scans from the top of the column to the bottom therefore showing the progression of root growth.

Video S2. DR5:Iuciferase luminescence and DR5rev:3xVENUS-N7 fluorescence in the LR repression experiments viewed using time-lapse imaging, related to Figure 4.

(First video) 4 or $5 \mathrm{dpg}$ Arabidopsis DR5:luciferase seedlings grown on standard media were transferred to standard media with DMSO (left panel) or $30 \mu \mathrm{M}$ ABA (right panel) and imaged every 10 minutes up to 18 hours in a dark imaging chamber. (Second video) 4 or 5 dpg Arabidopsis DR5rev:3xVENUSN7 seedlings grown on standard media were transferred directly on growth medium with DMSO (left panel) or $30 \mu \mathrm{M}$ ABA (right panel) and imaged every 2 minutes up to 24 hours. 

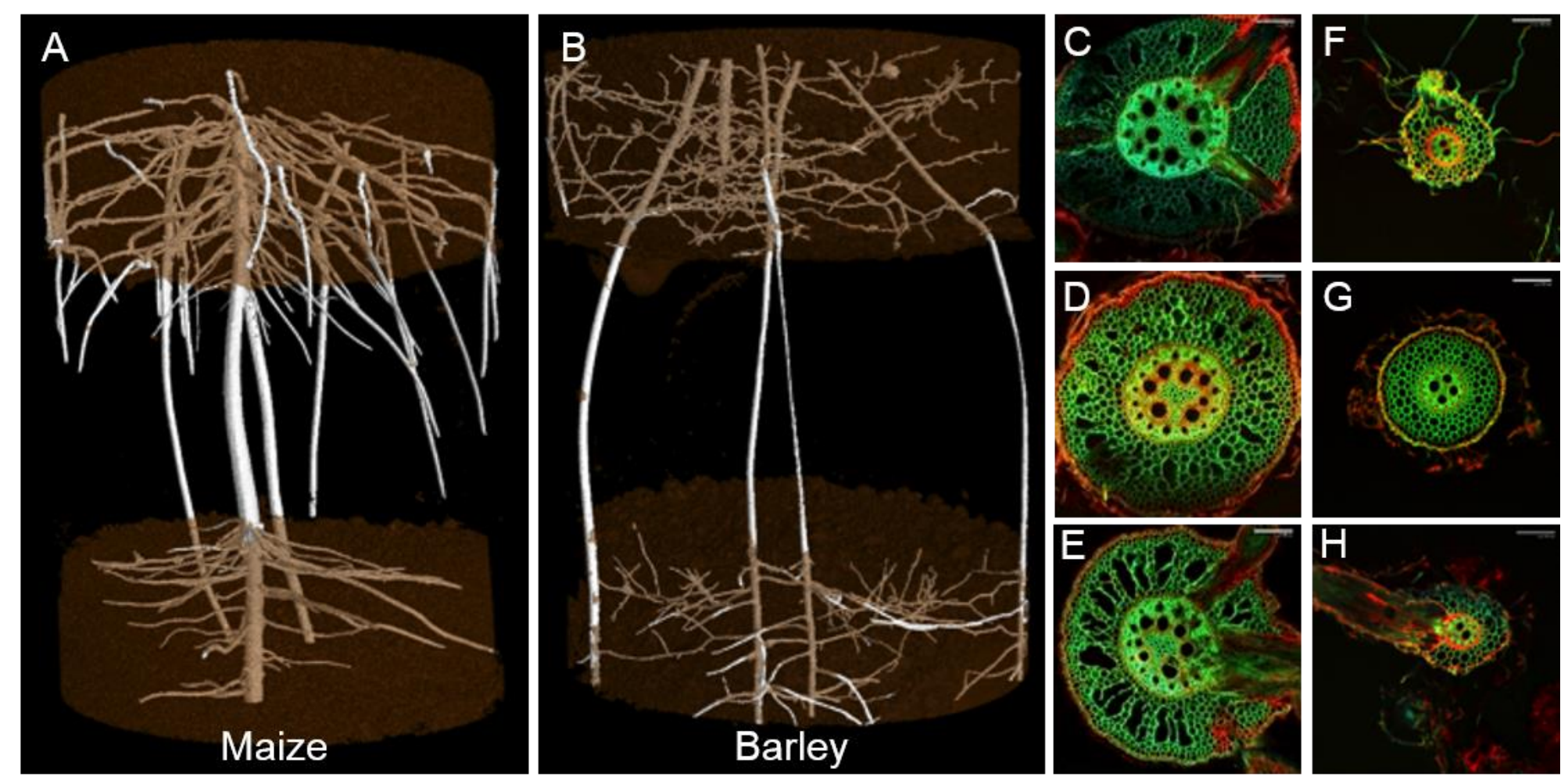

Figure 1 
A \begin{tabular}{cccccc}
\hline & Control & & Transient water deficit \\
\hline
\end{tabular}

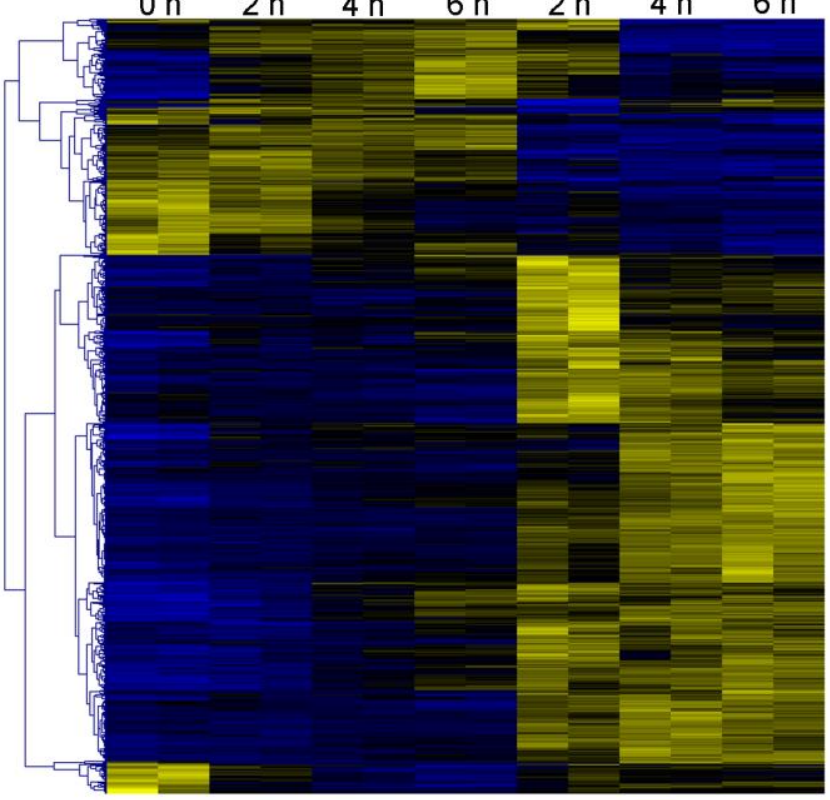

B $-2.33$
C

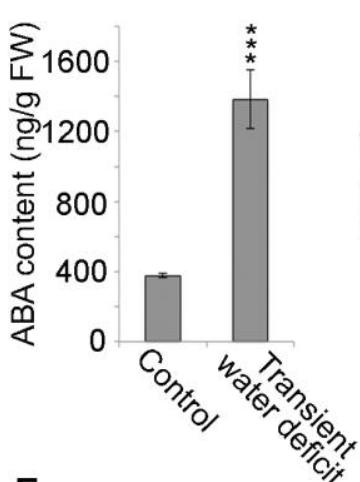

E

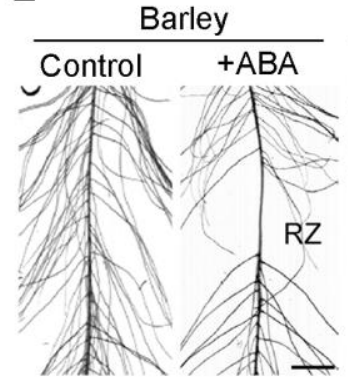

D

1.6

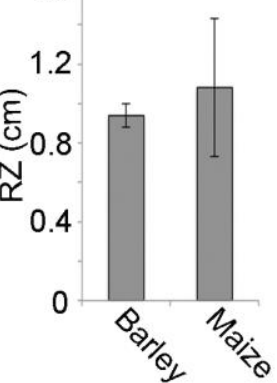

Maize

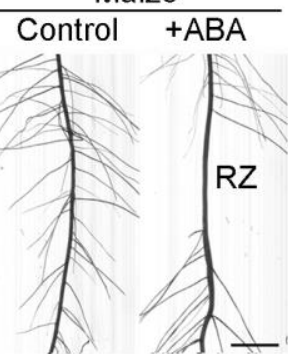

Regulation of salicylic acid metabolic process [GO:0010337]

Maintenance of seed dormancy by absisic acid [Go:0098755] Jasmonic acid and ethylene-dependent [GO:0009861]
systemic resistance Abscisic acid-activated signaling pathway [GO:0009738] Cellular response to abscisic acid stimulus [GO:0071215] Response to cytokinin [GO:0009735] Response to salicylic acid [GO:0009751] Jasmonic acid metabolic process [GO:0009694] Salicylic acid biosynthetic process [GO:0009697] Regulation of salicylic acid biosynthetic process [GO:0080142] Positive regulation of salicylic acid mediated [GO:0080151]
signaling pathway Negative regulation of brassinc signaling pathway

Jasmonic acid biosynthetic process [GO:0009695]

Salicylic acid metabolic process [GO:0009696] Auxin-activated signaling pathway [GO:0009734]

Salicylic acid catabolic process [GO:0046244] Cellular response to auxin stimulus [GO:0071365] Response to auxin [GO:0009733] Regulation of jasmonic acid mediated
signaling pathway [GO:2000022] se to abscisic acid [GO:0009737] Response to abscisic acid [GO:0009737] Cellular response to jasmonic acid stimulus [GO:0071395] Jasmonic acid mediated signaling pathway [GO:0009867]

Response to jasmonic acid [GO:0009753]

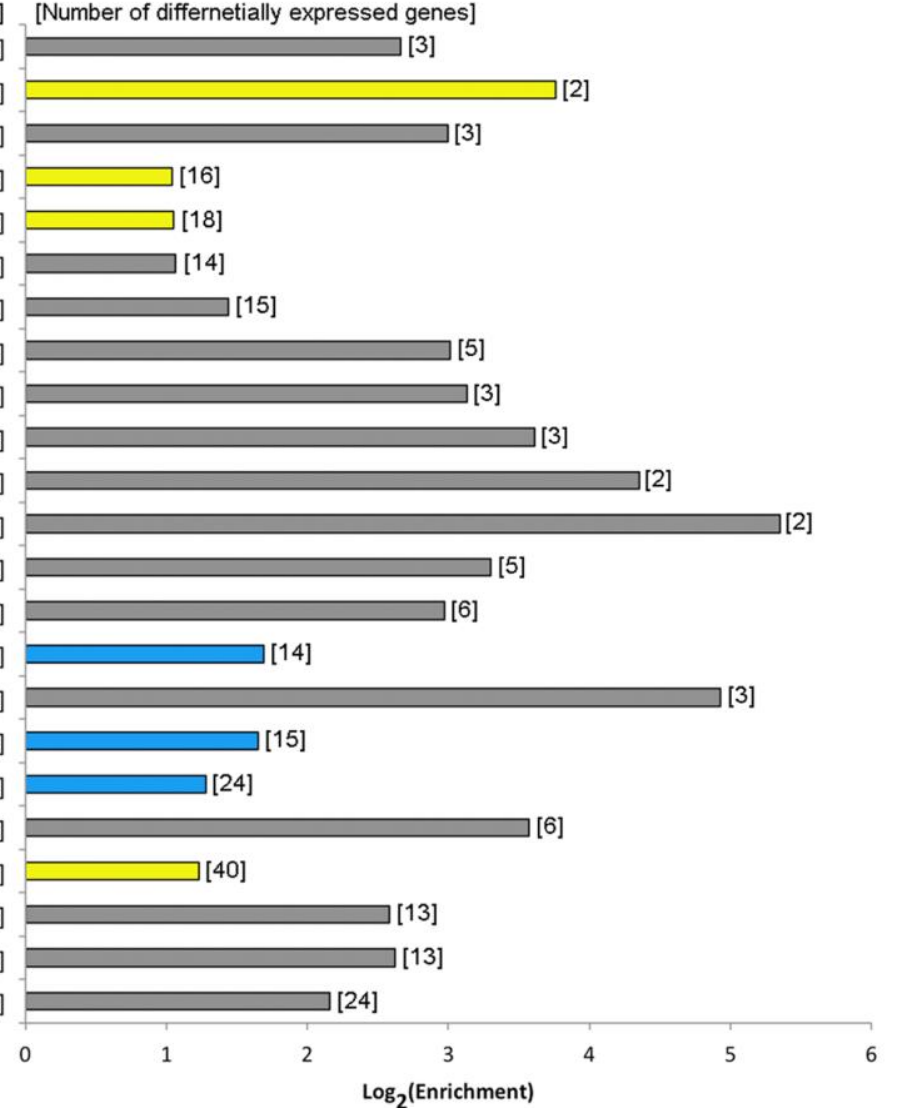

Figure 2 
A
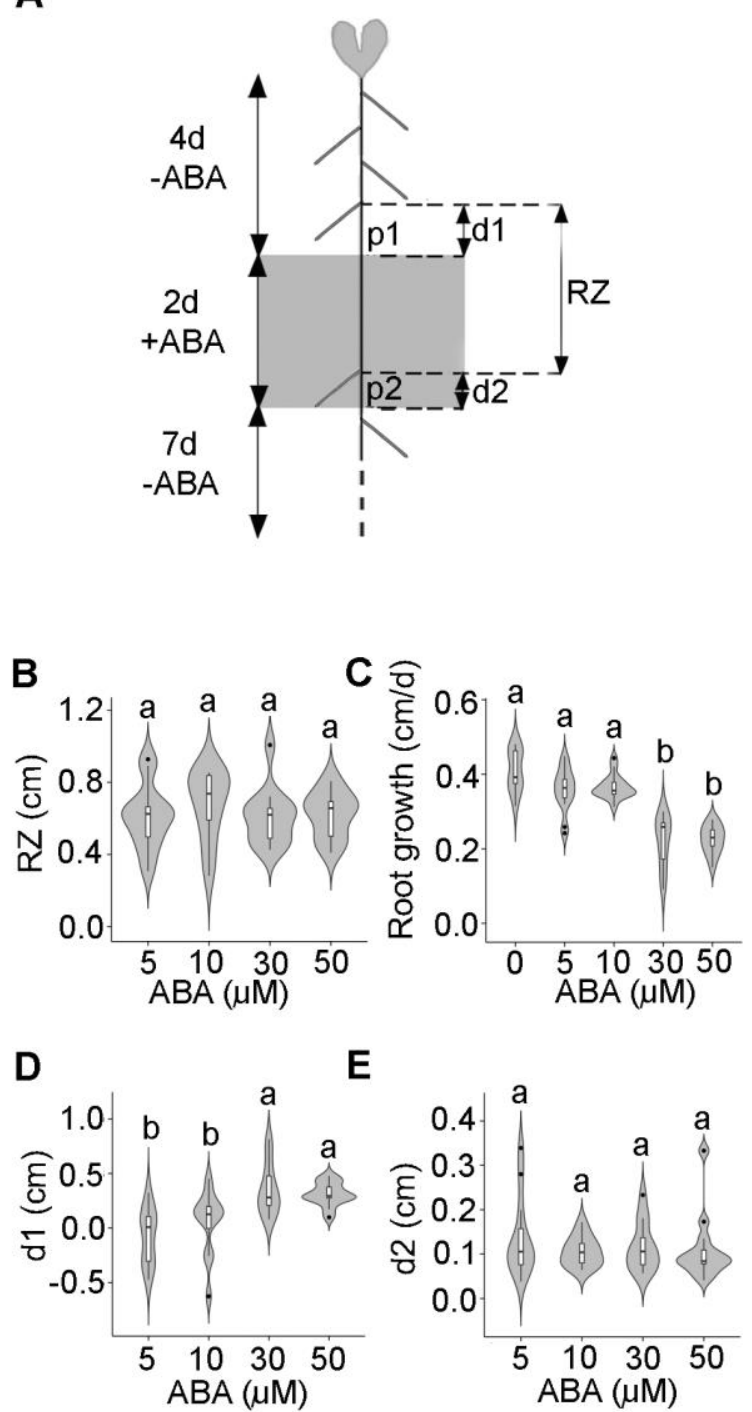

E

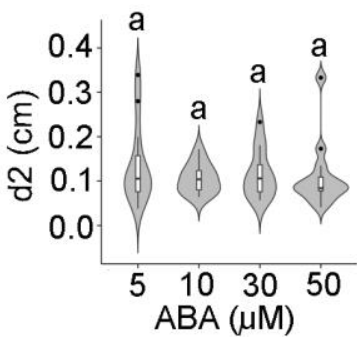

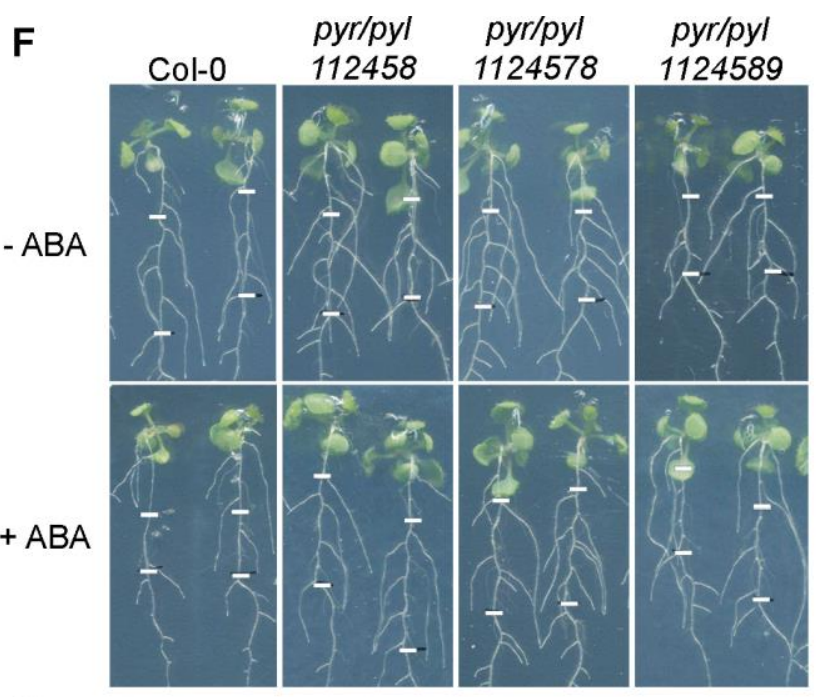

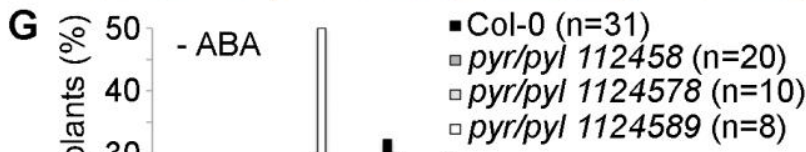

- pyr/pyl $1124589(\mathrm{n}=8)$

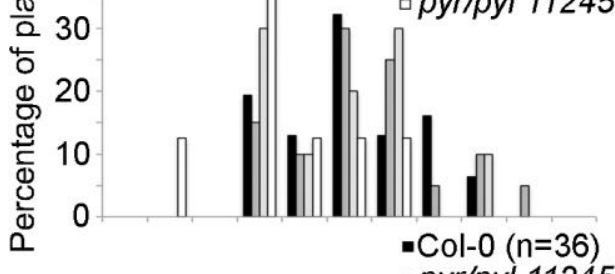

H $50+A B A$

pyr/pyl $112458(\mathrm{n}=31)$

口pyr/pyl $1124578(\mathrm{n}=29)$

口pyr/pyl $1124589(\mathrm{n}=10)$

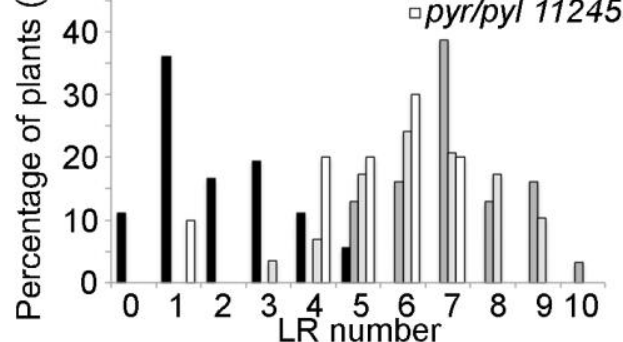

Figure 3 


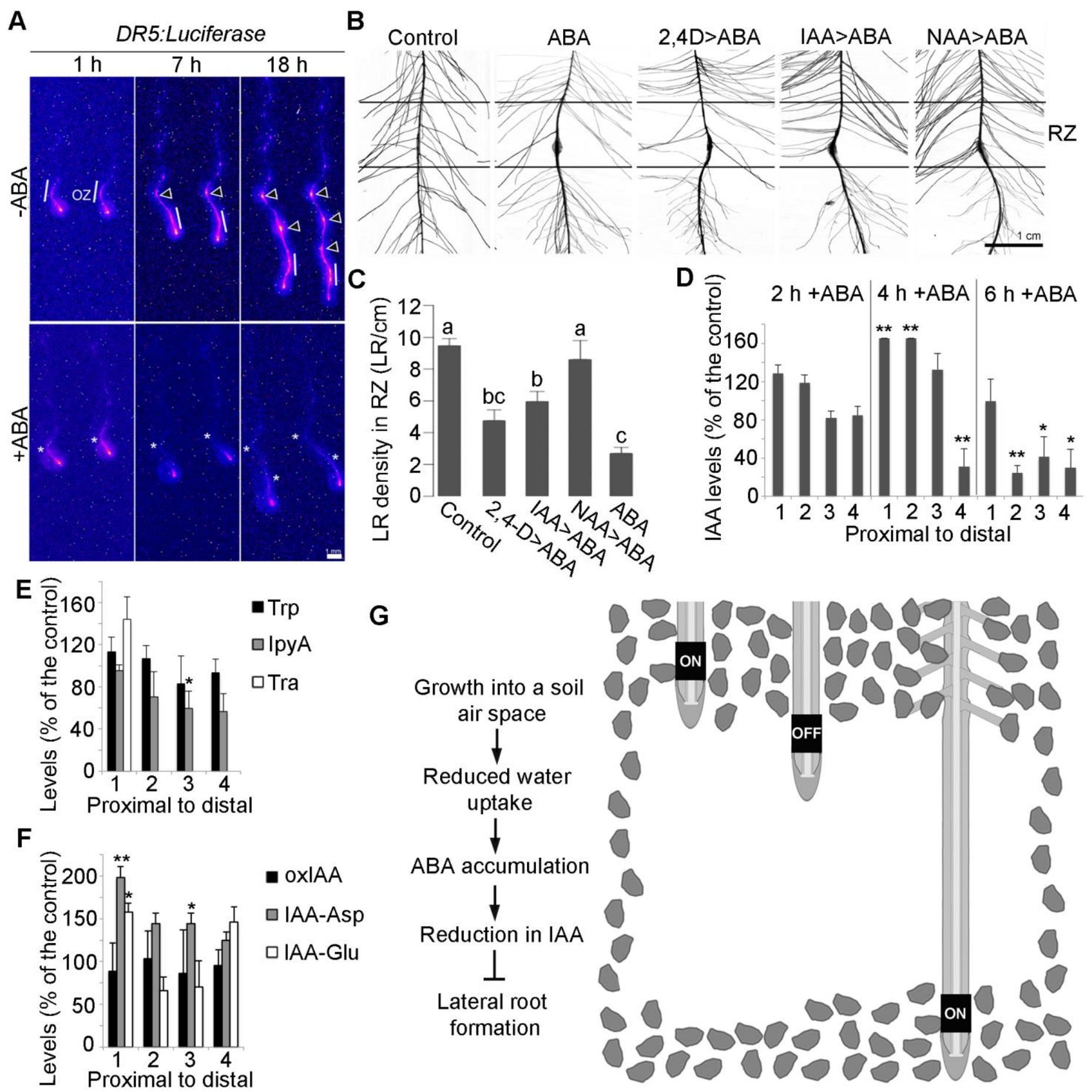

Figure 4 

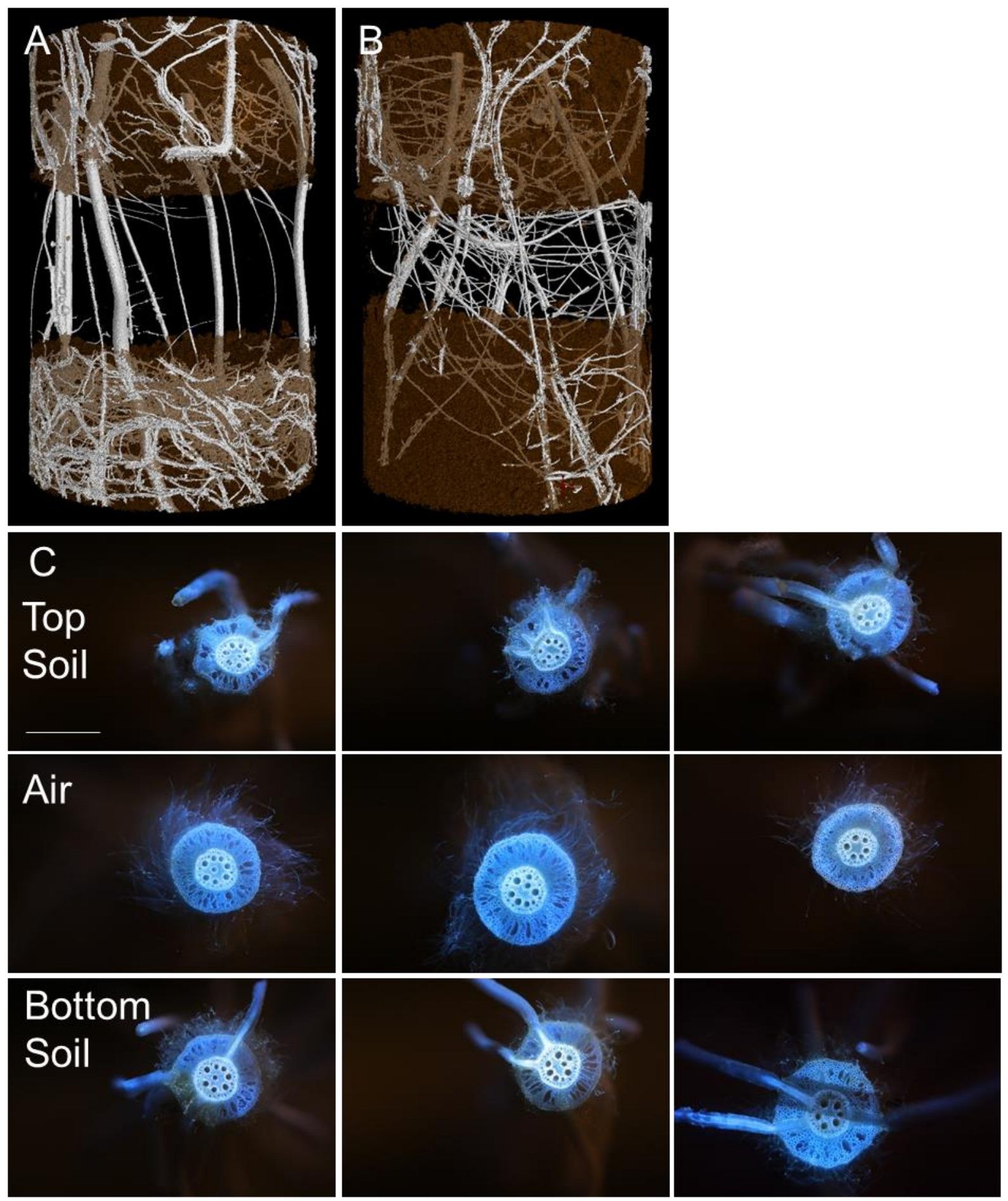

Figure S1. The xerobranching response is not observed when soil columns are submerged under water and laser ablation tomography confirms no primordia emerge when roots pass through air gaps, related to Figure 1.

(A and B) X-ray CT images of 16 day old maize (variety Emmerson). Root tissue is falsecolored in white and soil is false-colored in brown. (A) Maize grown through a $2 \mathrm{~cm}$ air gap show clear repression of branching $(n=3)$. (B) Maize grown through a $2 \mathrm{~cm}$ water filled gap, the whole column was submerged in water, continued to branch $(n=3)$. (C) Representative images created with laser ablation tomography (LAT) of cross sections taken from maize (B73) primary roots in in the top section of the soil, air gap and bottom section of soil from 3 different plants. Sample size of 187 images from 4 maize plants. Scale bar=1 $\mathrm{mm}$. 
A

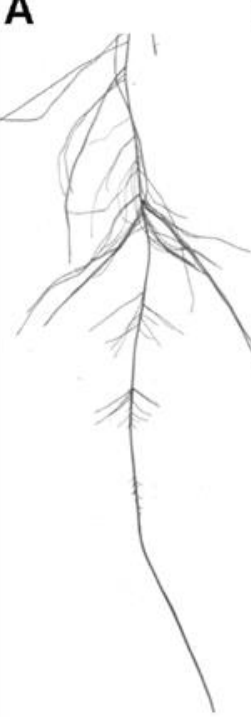

Barley cv. B83

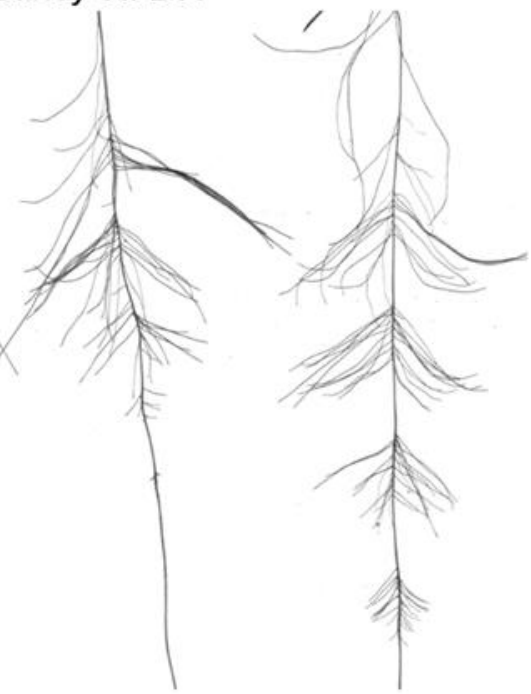

Barley cv. Derkado

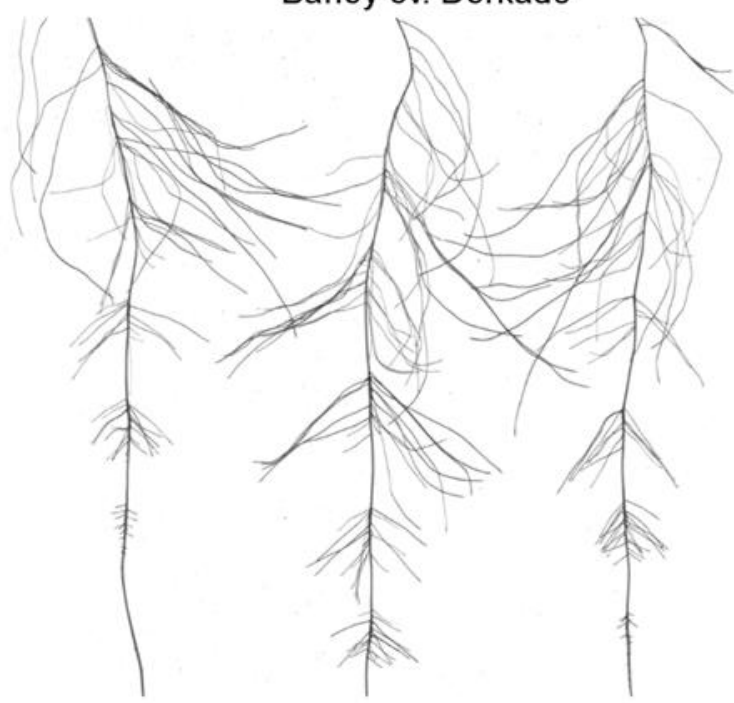

1

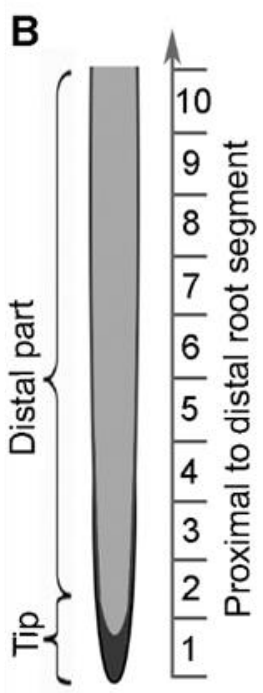

C

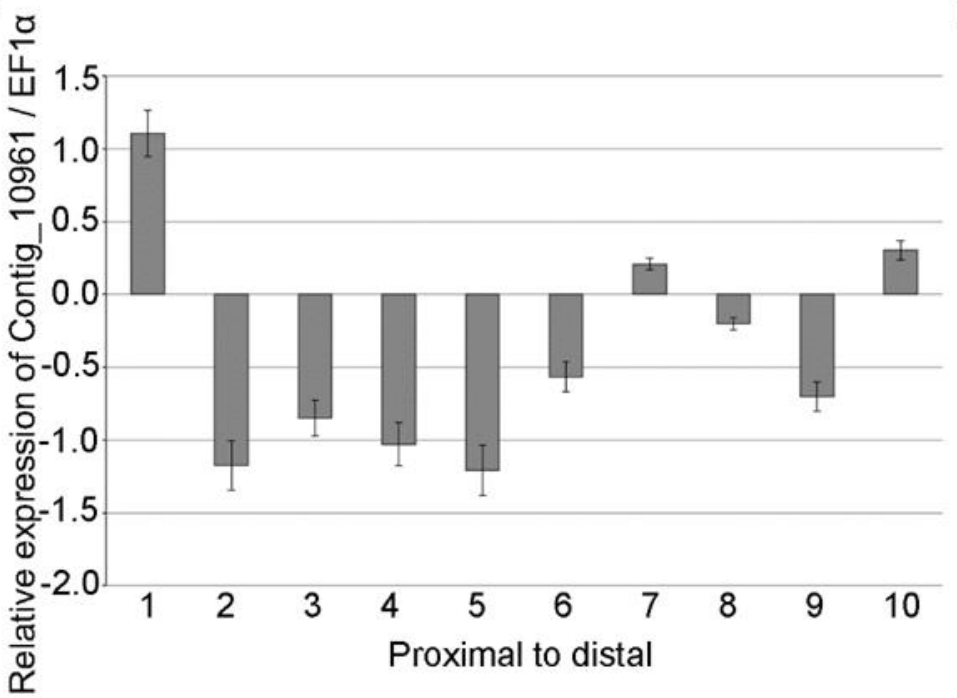

D

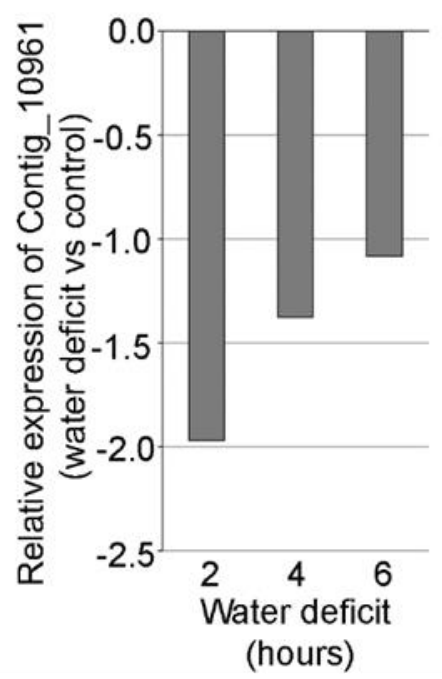

Figure S2. The branching response of barley cv. B83 and cv. Derkado in water deficit assay and qRT-PCR validation of water deficit microarray, related to Figure 2.

(A) Successive xerobranching responses in barley seedlings (left: B83, $n=3$; right: Derkado, $n=3$ ) subjected to alternating 16 hour water supply (day) and 8 hour water deficit (night) during four days. Picture acquired 8 days after sowing, proximal root segments only. Scale: $1 \mathrm{~cm}$. (B and C) Sampling design and results of the qPCR validation of the bZIP transcription factor (termed Contig_10961) expression in root segments after exposure of the root system to 2 hour long water deficit. We sampled ten successive root segments (from 1 to 10), $2 \mathrm{~mm}$ long each, along the $20 \mathrm{~mm}$ long root apical segments. The expression results are expressed relative to the control EF1a using the $\Delta \Delta C T$ method $(n=2)$ (D) Relative expression of Contig_10961 in root segments exposed to water deficit in the microarray experiment: expression values of Contig_10961 (drought vs control) are based on the barley microarray datasets (this paper), in which root systems were exposed to 2,4 and 6 hour long water deficit. The region sampled in the microarray experiment corresponds to positions 3-4 (2h), 4-5 (4h) and 5-6 (6h) of the qPCR validation in B and C. 

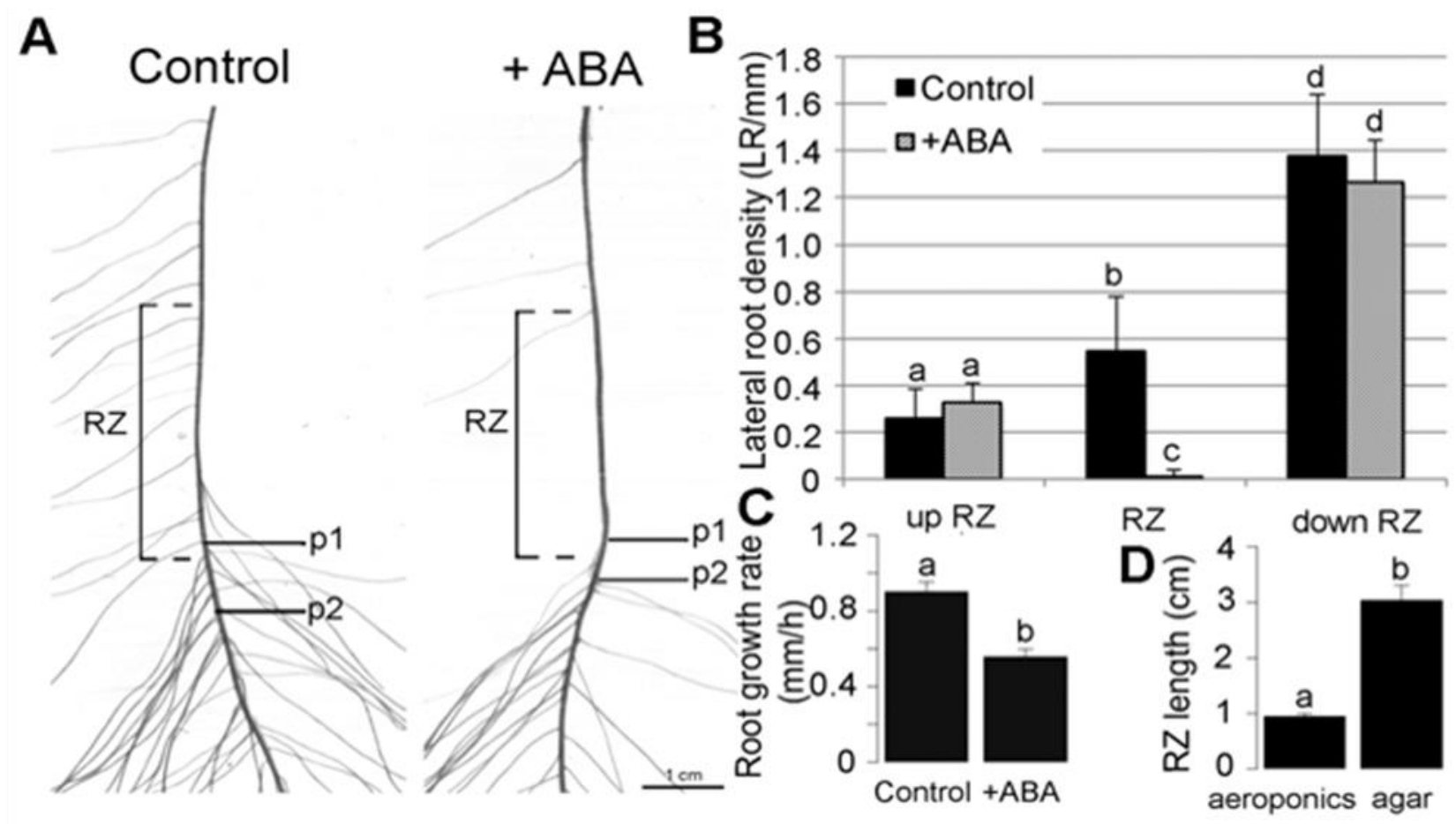

Figure S3. Effect of transient ABA treatment on agar plates on barley root branching pattern, related to Figure 2.

(A) Branching pattern of barley (B83) roots in transient ABA treatment assay on agar plates. 3 day old plants grown on filter paper were transferred on agar plates with DMSO (Control) or $50 \mu \mathrm{M}$ ABA (+ABA) for 6 hours. Positions of root tips were marked on plates before and after the treatment, seedlings were imaged, transferred to aeroponics and after 7 days of growth, plants were imaged again. (B) Number of LRs in the repression zone (RZ) and in $1 \mathrm{~cm}$-long root segments above (up RZ) and below RZ (down RZ), and (C) estimated root growth rate during 6 hour long $A B A$ ( $n=$ 20 ) or DMSO (Control) treatments $(n=20)$ on agar plates. (D) Comparison of RZs formed during transient 6 hour long ABA treatments in aeroponics $(n=17)$ and on agar plates $(n=10)$. Bars are means $\pm \mathrm{Cl}$. Different letters indicate significant differences according to Tukey's HSD test after ANOVA. Bar $=1 \mathrm{~cm}$. 
A

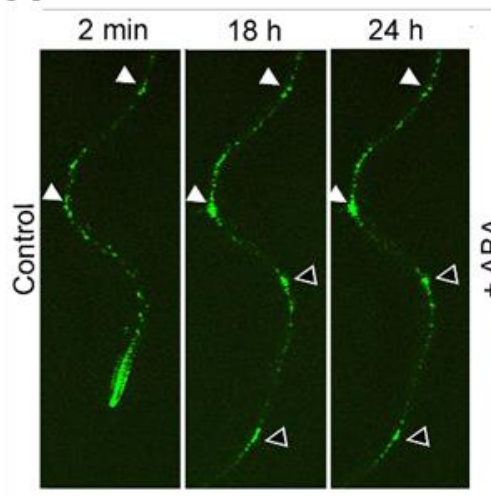

DR5:rev:3xVenus-N7

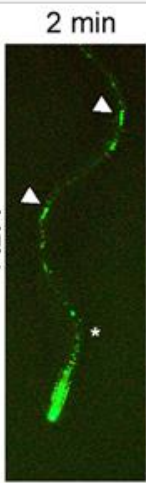

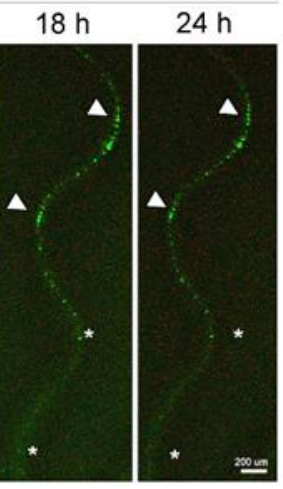

B

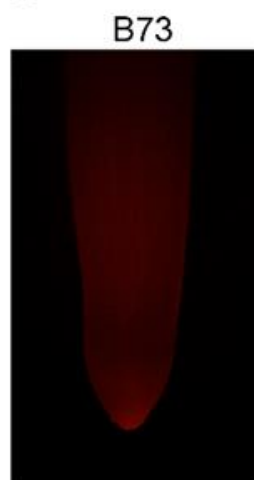

DR5:RFP in maize
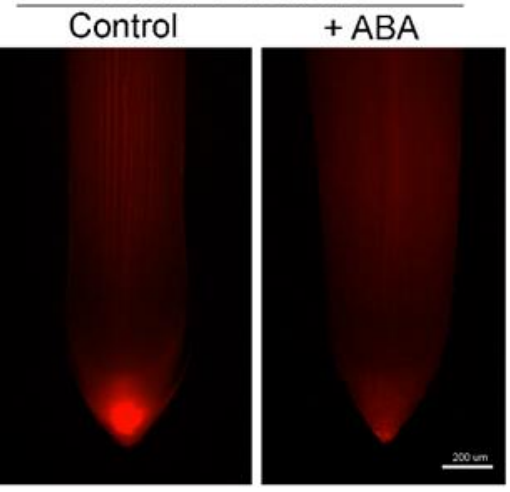

C

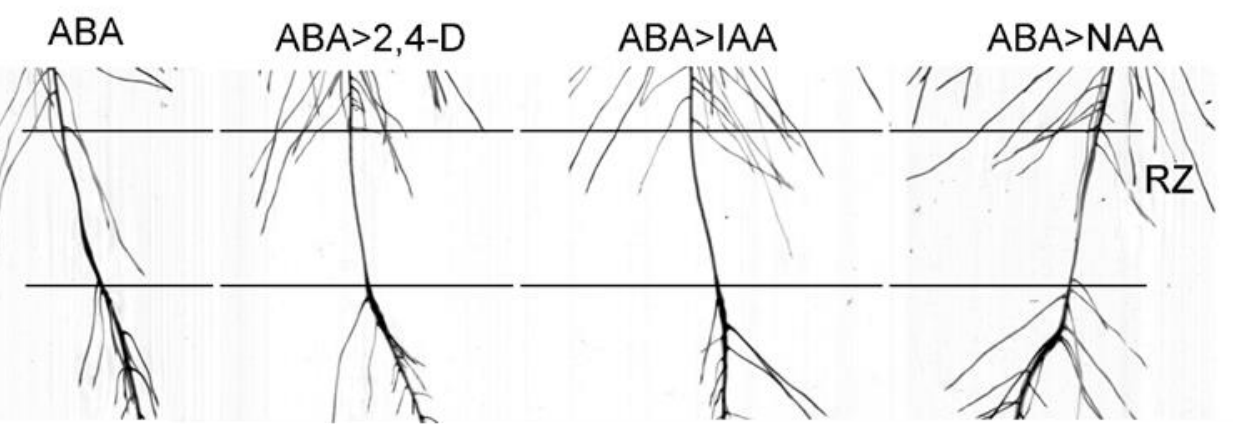

Figure S4. Transient ABA treatment alters the emergence of DR5 maxima in Arabidopsis and reduces DR5 fluorescence in maize. Applying auxin post ABA treatment does not restore LR branching in barley, related to Figure 4.

(A) Representative DR5rev:3XVENUS-N7 fluorescence in LR repression experiments viewed using time-lapse imaging. 4 or $5 \mathrm{dpg}$ Arabidopsis seedlings grown on standard

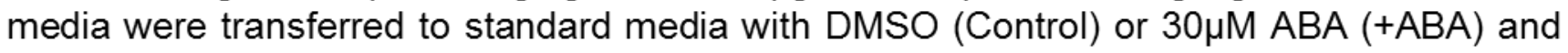
imaged every 2 minutes during 24 hour in a dark imaging chamber $(n=9)$. White arrowheads mark the position of DR5 maxima present at the time of transfer. Black-filled arrowheads indicate the position of DR5 maxima that appear during the time-lapse imaging. White stars mark the position where DR5 oscillation pulses should be present by virtue of their position along root. Bar $=200 \mu \mathrm{m}$. (B) DR5:RFP fluorescence in the root tip of maize (B73) in LR repression experiments. Fluorescence was captured after 4 hours of DMSO (Control) $(n=7)$ or $50 \mu \mathrm{M}$ ABA application $(n=8)$ in aeroponics. Wild-type $B 73$ was used as a background control. Bar=200 $\mu \mathrm{m}$. (C) Effect of auxin post-treatment on ABA mediated LR repression in barely. Branching pattern of barley (B83) roots in auxin complementation assay. Plants were

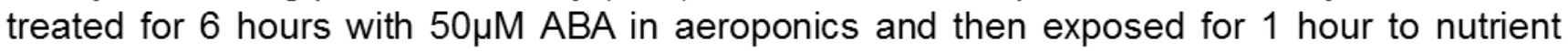
solution $(n=6)$, or to $75 \mu M$ of $2,4-D(n=8)$, IAA $(n=9)$ or NAA $(n=8)$. Roots were imaged after 7 days of growth. 
